\title{
Quiver indices and Abelianization from Jeffrey-Kirwan residues
}

\author{
Guillaume Beaujard, ${ }^{a}$ Swapnamay Mondal ${ }^{b}$ and Boris Pioline ${ }^{a}$ \\ ${ }^{a}$ Laboratoire de Physique Théorique et Hautes Energies (LPTHE), \\ UMR 7589 CNRS-Sorbonne Université, Campus Pierre et Marie Curie, \\ 4 place Jussieu, F-75005 Paris, France \\ ${ }^{b}$ International Centre for Theoretical Sciences, \\ Tata Institute of Fundamental Research, Shivakote, \\ Hesaraghatta, Bangalore 560089, India \\ E-mail: beaujard@lpthe.jussieu.fr, swapnamay.mondal@icts.res.in, \\ pioline@lpthe.jussieu.fr
}

ABSTRACT: In quiver quantum mechanics with 4 supercharges, supersymmetric ground states are known to be in one-to-one correspondence with Dolbeault cohomology classes on the moduli space of stable quiver representations. Using supersymmetric localization, the refined Witten index can be expressed as a residue integral with a specific contour prescription, originally due to Jeffrey and Kirwan, depending on the stability parameters. On the other hand, the physical picture of quiver quantum mechanics describing interactions of BPS black holes predicts that the refined Witten index of a non-Abelian quiver can be expressed as a sum of indices for Abelian quivers, weighted by 'single-centered invariants'. In the case of quivers without oriented loops, we show that this decomposition naturally arises from the residue formula, as a consequence of applying the Cauchy-Bose identity to the vector multiplet contributions. For quivers with loops, the same procedure produces a natural decomposition of the single-centered invariants, which remains to be elucidated. In the process, we clarify some under-appreciated aspects of the localization formula. Part of the results reported herein have been obtained by implementing the Jeffrey-Kirwan residue formula in a public Mathematica code.

KEYwords: Supersymmetric Gauge Theory, Black Holes in String Theory, M(atrix) Theories

ARXiv EPrint: 1907.01354 


\section{Contents}

1 Introduction 1

2 A brief review of the Coulomb branch formula for quivers 4

2.1 The Coulomb branch formula 6

2.2 The MPS formula 8

3 Indices from residues $\quad 8$

3.1 Review of the JK residue prescription 11

$\begin{array}{lll}3.2 & \text { Residues and fixed points } & 12\end{array}$

$\begin{array}{lll}3.3 & \text { Index in attractor chamber } & 13\end{array}$

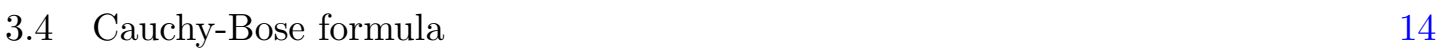

4 Abelian quivers $\quad \mathbf{1 5}$

4.1 Abelian quivers without loops 15

$\begin{array}{ll}\text { 4.2 Abelian quivers with unoriented loops } & 17\end{array}$

$\begin{array}{lll}\text { 4.2.1 Three node quiver } & 17\end{array}$

$\begin{array}{lll}4.2 .2 & \text { Four node quiver } & 18\end{array}$

$\begin{array}{lll}4.3 & \text { Abelian quivers with oriented loops } & 19\end{array}$

5 Non-Abelian quivers $\quad 23$

5.1 Kronecker quiver with rank $(N, 1) \quad 23$

$\begin{array}{lll}5.2 & \text { Star quivers } & 25\end{array}$

5.3 Kronecker quiver with rank $\left(N_{1}, N_{2}\right) \quad 28$

$\begin{array}{lll}5.3 .1 & \text { Rank }(2,3) & 28\end{array}$

5.3.2 Rank $(2,2) \quad 31$

5.4 Non-abelian quivers with oriented loops 33

A Implementation of the residue formula in CoulombHiggs.m 36

\section{Introduction}

In four-dimensional quantum field theories or string vacua with 8 supercharges, BPS states with mutually non-local electromagnetic charges $\gamma_{i}(i=1 \ldots n)$, can generically form supersymmetric bound states, or BPS molecules, which are stable in some domain in moduli space. These bound states can be understood as multi-centered black hole solutions to $\mathcal{N}=2$ supergravity in the string theory context $[1,2]$, or as multi-centered Dirac-Julia-Zee dyons in the low energy description of $\mathcal{N}=2$ gauge theories on the Coulomb branch [3, 4]. 
In the non-relativistic limit, the interactions between the centers can be described by a supersymmetric quantum mechanics of $n$ particles in $\mathbb{R}^{3}$, interacting through electromagnetic, scalar exchange and possibly gravitational forces [5-9].

Alternatively, in a regime where the BPS constituents can be represented by D-branes, their dynamics is well described by a supersymmetric gauge theory in $0+1$ dimension $[7,10$ 15] with a unitary gauge group $\mathrm{U}\left(N_{a}\right)$ for each species of charge $\alpha_{a}$ occurring among the $\gamma_{i}$ 's with multiplicity $N_{a}$, and with $\kappa_{a b}$ chiral multiplets $\phi_{a b, A}, A=1, \ldots \kappa_{a b}$ in the bifundamental representation $\left(N_{a}, \bar{N}_{b}\right)$ whenever the charges $\alpha_{a}, \alpha_{b}$ have non-negative DiracSchwinger-Zwanziger product $\kappa_{a b}=\left\langle\alpha_{a}, \alpha_{b}\right\rangle$. The field content is graphically represented by a quiver $Q$ with vertices $V_{a} \in \mathcal{V}_{Q}$ associated to the gauge groups $\mathrm{U}\left(N_{a}\right)$ and with arrows $e_{a b, A} \in \mathcal{A}_{Q}$ associated to the chiral multiplets. We shall refer to the corresponding quantum mechanics with 4 supercharges as quiver quantum mechanics. The dimension vector $\left(N_{1}, \ldots, N_{K}\right)$ is identified with the total electromagnetic charge $\gamma=\sum_{a=1}^{K} N_{a} \alpha_{a}=\sum_{i=1}^{n} \gamma_{i}$ of the BPS molecule.

The dependence of the dynamics on the gauge or string theory moduli, which we denote collectively by $z$, is encoded through Fayet-Iliopoulos parameters $\left\{\zeta_{a}(z)\right\}$ associated to each factor in the gauge group, as well as through a gauge invariant superpotential $\mathcal{W}(\phi, z)$ whenever the quiver has oriented closed loops. BPS bound states correspond to supersymmetric ground states of this quantum mechanics [7]. In the simplest case where the ranks $N_{a}$ are coprime and the superpotential is generic, these BPS bound states are in one-to-one correspondence with Dolbeault cohomology classes on the moduli space $\mathcal{M}_{Q} \equiv \mathcal{M}_{Q}\left(\left\{N_{a}, \zeta_{a}\right\}\right)$ of stable representations, an algebraic variety of central interest in representation theory (see e.g. [16]). In particular, the refined Witten index $\Omega(\gamma, z, y)=$ $\operatorname{Tr}(-1)^{2 J_{3}} y^{2\left(I_{3}+J_{3}\right)}$ in the supersymmetric quantum mechanics (where $J_{3}, I_{3}$ are Cartan generators of the R-symmetry group $\mathrm{SU}(2)_{L} \times S U_{2}(R)$ ) coincides (up to a simple prefactor) with the $\chi$-genus of the moduli space $\mathcal{M}_{Q}[17,18]$,

$$
\left.\Omega(\gamma, z, y):=\chi_{Q}\left(\left\{N_{a}, \zeta_{a}\right)\right\}, y\right):=\sum_{p, q=0}^{d}(-1)^{p+q-d} y^{2 p-d} h_{p, q}\left(\mathcal{M}_{Q}\right)
$$

which in turn is the specialization at $t=y$ of the Hodge polynomial

$$
\Omega(\gamma, z, y, t):=\sum_{p, q=0}^{d}(-y)^{p+q-d} t^{p-q} h_{p, q}\left(\mathcal{M}_{Q}\right)
$$

Here $d$ is the complex dimension of $\mathcal{M}_{Q}$ and $h_{p, q}$ are the Hodge numbers, such that $\chi_{Q}$ coincides with $(-1)^{d}$ times the Euler number $\chi\left(\mathcal{M}_{Q}\right)$ when $y=1$. While the Hodge numbers are insensitive to the choice of superpotential away from complex codimensionone loci, they depend crucially on the stability parameters $\zeta_{a}$, and may jump across real codimension-one known as 'walls of marginal stability', where $\sum_{a=1}^{K} n_{a} \zeta_{a}=0$ for some positive integers $n_{a}$. The jump is given by a universal wall-crossing formula [19-21] which has a transparent physical interpretation in terms of the (dis)appearance of multi-centered black hole solutions [22-24]. 
In general, the direct computation of the Hodge numbers $h_{p, q}\left(\mathcal{M}_{Q}\right)$ is a difficult task. In the absence of oriented loops and for primitive dimension vector, $\mathcal{M}_{Q}$ is a pure projective variety, whose Poincaré polynomial, which can be computed by counting points over finite fields [25], and moreover the cohomology is supported in degree $(p, p)$ [26], so the $\chi$-genus coincides with the Poincaré polynomial. Recently, building on previous work on twodimensional gauge linear sigma models $[27,28],{ }^{1}$ the $\chi$-genus (1.1) for any quiver was computed using the method of supersymmetric localization [31-33], which is closely related to the Atiyah-Bott Lefschetz fixed point theorem. The result is expressed as a suitable combination of residues of a rational function $Z_{Q}\left(\left\{u_{a, i}\right\}\right)$ of the (complexified) adjoint scalars $u_{a, i}, i=1 \ldots N_{a}$ for each gauge group $\mathrm{U}\left(N_{a}\right)$, restricted to the Cartan torus. For a fixed value of the stability parameters $\left\{\zeta_{a}\right\}$, the contributing poles and the order of integration around each of them are determined according to a prescription originally due to Jeffrey and Kirwan [34]. Different integration contours arise in different chambers, such that discontinuities across walls of marginal stability are consistent with the wall-crossing formula [31]. Despite being completely algorithmic, the residue formula becomes quickly unwieldy even for moderate ranks, due to a proliferation of possible poles and orders of integration.

Based on the interpretation in terms of a multi-centered configuration of $n$ BPS black holes with charges $\gamma_{i}$, it was suggested in [36], and further elaborated in [17,37,38], that the Witten index (or $\chi$-genus) of a non-Abelian quiver $Q$ with gauge group $\prod_{a=1}^{K} \mathrm{U}\left(N_{a}\right)$ could be decomposed as a sum of Witten indices $\chi_{Q\left(\left\{\gamma_{i}\right\}\right)}$ for a family of Abelian quivers $Q\left(\left\{\gamma_{i}\right\}\right)$ associated to all decompositions of the dimension vector $\gamma=\sum_{a=1}^{K} N_{a} \alpha_{a}$ into a sum $\gamma=\sum_{i=1}^{m} \gamma_{i}$ where each $\gamma_{i}=\sum_{a=1}^{K} n_{i, a} \alpha_{a}$ is itself a linear combination of the basis vectors associated to the nodes of the original quiver, with positive integer coefficients $n_{a, i}$. The general formula is known as the 'Coulomb branch formula' from $[17,36]$ (see section 2 below for a precise statement) and the coefficients appearing in front of each $\chi_{Q\left(\left\{\gamma_{i}\right\}\right)}$ involve a new set of invariants $\bar{\Omega}_{\mathrm{S}}\left(\gamma_{i}\right)$ known as 'intrinsic Higgs invariants', 'quiver invariants' or 'single-centered invariants' $[17,18,39,40]$, which are independent of the stability conditions, and conjecturally depend only on the variable $t$ conjugate to $p-q$ in (1.2), but not on $y$ [17]. These invariants are currently defined in an indirect, recursive way (see [41] for a concise explanation of the Coulomb branch formula). This conjecture, if true, gives a powerful way of obtaining the the full Hodge polynomial from the knowledge of $\chi$-genus of $Q$ and of its subquivers.

For quivers without oriented loops and primitive dimension vector however, the singlecentered invariants are known to have support only on multiples of the basic vectors $\gamma_{i}=$ $\ell \alpha_{a}$, in which case they are simply given by $\bar{\Omega}_{\mathrm{S}}\left(\ell \alpha_{a}\right)=(y-1 / y) /\left[\ell\left(y^{\ell}-y^{-\ell}\right)\right]$ for any vertex $V_{a} \in \mathcal{V}_{Q}$. The Coulomb branch formula (sometimes known as the MPS formula in this restricted setting) then becomes completely explicit, and reduces to a sum of the $\chi$-genera for the Abelian quivers $Q\left(\left\{\gamma_{i}\right\}\right)$, with simple combinatorics coefficients. This formula was first derived in [24, appendix D] based on the Reineke formula [25] for quivers without loops, and put on a rigorous mathematical ground in [42].

\footnotetext{
${ }^{1}$ This type of computation was pioneered in $[29,30]$.
} 
Our main goal in this paper will be to derive the Abelianization formula for quivers without oriented loops (where the MPS formula is already known to hold) by manipulating the residue formula of [31]. The key idea is to use the Cauchy-Bose formula

$$
\operatorname{det} \frac{1}{\sinh \left(\mu_{i}-\nu_{j}\right)}=\frac{\prod_{i<j} \sinh \left(\mu_{i}-\mu_{j}\right) \sinh \left(\nu_{j}-\nu_{i}\right)}{\prod_{i, j} \sinh \left(\mu_{i}-\nu_{j}\right)}
$$

to decompose the vector multiplet determinant for a given $\mathrm{U}\left(N_{a}\right)$ gauge group into a sum over conjugacy classes in the permutation group $S_{N_{a}}$, which are labelled by partitions $\lambda=$ $\sum_{\ell \geq 1} \ell N_{a, \ell}$. Applying (1.3) to each $\mathrm{U}\left(N_{a}\right)$ factor, leads to a sum over all decompositions of the dimension vector $\gamma=\sum_{i} \gamma_{i}$ where the $\gamma_{i}$ 's are positive linear combinations of the vectors $\ell \alpha_{a}$. We shall see that the contribution $\chi_{Q}^{\lambda}$ of permutations ${ }^{2}$ in the conjugacy class $\lambda$ associated to a decomposition $\gamma=\sum_{\ell, a} n_{\ell, a}\left(\ell \alpha_{a}\right)$ reproduce the contribution of the Abelian quiver $Q\left(\left\{\gamma_{i}\right\}\right)$ to the MPS formula for $\chi_{Q}$. We note that the formula (1.3) (or rather its rational limit) was used in a similar context in the computation of the index of $\mathcal{N}=4$ SYM in [30], and as a tool to construct grand canonical partition functions for matrix models in [30, 43, 44]. An elliptic version (3.13) of this equation also allows to implement a similar Abelianization process for computing the elliptic genus two-dimensional gauge linear sigma models [28]. Finally, we note that the Abelianization formula for quivers was also investigated using toric geometry in [45], where a similar but less manipulation of the vector multiplet determinant was used.

The outline of this work is as follows. In section 2, we recall basic definitions and facts about quiver moduli spaces, and briefly review the Coulomb branch formula and the MPS formula. In section 3, we review the residue prescription of $[28,31]$ for computing the $\chi$-genus $\chi_{Q}\left(\left\{N_{a}, \zeta_{a}\right\}\right)$ of the quiver moduli space $\mathcal{M}_{Q}$, recall the relation with the AtiyahBott fixed point theorem, examine its value in the attractor chamber, and introduce the Cauchy-Bose formula as a useful way of decomposing the index into a sum over partitions of the dimension vector. In section 4 , we apply the residue prescription to various examples of Abelian quivers with or without oriented loop, clarifying the origin of the stability condition for flags contributing to the residue formula. In section 5 , we consider various examples of non-Abelian quivers, and explain the origin of the various terms in the Coulomb branch or MPS formula in terms of conjugacy classes in the Cauchy-Bose formula. In appendix A, we indicate how to reproduce some our results by using the mathematica package CoulombHiggs.m developed by the last-named author, which was originally released along with [37], and has been extended to include the Jeffrey-Kirwan residue formula and other functionalities.

\section{A brief review of the Coulomb branch formula for quivers}

Let $Q$ be a quiver with vertices $V_{a} \in \mathcal{V}_{Q}(a=1 \ldots K)$, arrows $e_{a b, A} \in \mathcal{A}_{Q}$. We assume that there are no arrows from one vertex to itself, and that all arrows $e_{a b, A}$ between vertices $V_{a}$ and $V_{b}$ point in the same direction. We denote by $\kappa_{a b}$ the number of arrows from $V_{a}$ to $V_{b}$,

\footnotetext{
${ }^{2}$ In fact, all permutations in the same conjugacy class turn out to contribute equally, naturally leading to the Boltzmann symmetry factor in the MPS formula.
} 
and by $-\kappa_{a b}$ the number of arrows from $V_{b}$ to $V_{a}$, so that $\kappa_{a b}$ is an antisymmetric matrix with integer entries. Let $\gamma=\left(N_{1}, \ldots, N_{K}\right)$ be a vector of non-negative integers, known as the dimension vector. The quiver quantum mechanics associated to $(Q, \gamma)$ is a $0+1$ dimensional gauge theory with four supercharges [7]. It includes vector multiplets for the gauge group $G=\prod_{a=1}^{K} \mathrm{U}\left(N_{a}\right)$ and $\left|\alpha_{a b}\right|$ chiral multiplets transforming in the bifundamental representation $\left(N_{a}, \bar{N}_{b}\right)$ if $\alpha_{a b}>0$, or its complex conjugate $\left(\bar{N}_{a}, N_{b}\right)$ if $\alpha_{a b}<0$. We shall denote the bosonic component of these chiral multiplets by $\phi_{a b, A, s s^{\prime}}$, where $1 \leq A \leq\left|\alpha_{a b}\right|$, $1 \leq s \leq N_{a}, 1 \leq s^{\prime} \leq N_{b}$. When the quiver has oriented loops, the Lagrangian depends on a superpotential $\mathcal{W}(\phi)$, which is a sum of $G$-invariant monomials in the chiral multiplets $\phi_{a b, A, s s^{\prime}}$. In addition, the Lagrangian depends on a real vector $\zeta=\left(\zeta_{1}, \ldots, \zeta_{K}\right)$, known as the stability vector, whose entries are Fayet-Iliopoulos (FI) parameters for the U(1) center in each gauge group $\mathrm{U}\left(N_{a}\right)$. Since the diagonal $\mathrm{U}(1)$ action leaves all fields invariants, we may assume that $\sum_{a=1}^{K} N_{a} \zeta_{a}=0$. For the purpose of counting BPS states, the overall scale of the $\zeta_{a}$ 's is also irrelevant, so this vector can be viewed as a point in real projective space $\mathbb{R} \mathbb{P}^{K}$.

Semi-classically, the quiver quantum mechanics admits two branches of supersymmetric vacua. On the Higgs branch, the gauge symmetry is broken to the U(1) center by the vevs of the chiral multiplet scalars $\phi_{a b, A, s s^{\prime}}$, which are subject to the D and F-term relations

$$
\begin{array}{rlrl}
\sum_{b: \kappa_{a b}>0} \sum_{\substack{s^{\prime}=1 \ldots b \\
A=1 \ldots \kappa_{a b}}} \phi_{a b, A, s s^{\prime}}^{*} \phi_{a b, A, t s^{\prime}} & \\
-\sum_{b: \kappa_{a b}<0} \sum_{\substack{s^{\prime}=1 \ldots b \\
A=1 \ldots\left|\kappa_{a b}\right|}} \phi_{b a, A, s^{\prime} s}^{*} \phi_{b a, A, s^{\prime} t} & =\zeta_{a} \delta_{s t} & \forall a, s, t \\
\frac{\partial W}{\partial \phi_{a b, A, s s^{\prime}}} & =0 & \forall a, b, A, s, s^{\prime}
\end{array}
$$

where $1 \leq a \leq K, 1 \leq s, t \leq N_{a}$ in the first equation while $1 \leq a, b \leq K, 1 \leq s \leq N_{a}, 1 \leq$ $s^{\prime} \leq N_{b}, 1 \leq A \leq \kappa_{a b}$ in the second equation, whenever $\kappa_{a b}>0$. Classical supersymmetric vacua are in one-to-one correspondence with orbits of solutions to (2.1), (2.2) under the compact gauge group $G=\prod_{a=1}^{K} \mathrm{U}\left(N_{a}\right)$. Equivalently, they are in one-to-one correspondence with stable orbits of solutions of (2.2) under the action of the complexified gauge group $G_{\mathbb{C}}=\prod_{a=1}^{K} \operatorname{GL}\left(N_{a}, \mathbb{C}\right)$, where the stability condition is determined by the vector $\zeta$. The set $\mathcal{M}_{Q}$ of supersymmetric vacua thus coincides with the moduli space of stable quiver representations widely studied in mathematics (see e.g. [16, 46] for entry points in the vast literature on this subject). Quantum mechanically, BPS states on the Higgs branch are harmonic forms on $\mathcal{M}_{Q}$, or equivalently Dolbeault cohomology classes [7]. The group $\mathrm{SO}(3)$ associated to physical rotations in $\mathbb{R}^{3}$ acts on the cohomology of the Higgs branch via the Lefschetz action generated by contraction and wedge product with the natural Kähler form on $\mathcal{M}_{Q}$, induced from the flat Kähler form on the ambient space $\oplus_{e_{a b A} \in \mathcal{A}_{Q}} \mathbb{C}^{N_{a}} \otimes \mathbb{C}^{N_{b}}$.

On the Coulomb branch, the gauge symmetry is broken to the diagonal subgroup $\mathrm{U}(1)^{\sum_{a=1}^{K} N_{a}}$ and all chiral multiplets as well as off-diagonal vector multiplets are massive. After integrating out these degrees of freedom, the diagonal part $\vec{r}_{i}$ of the scalars in the 
vector multiplets must be solutions to Denef's equations [7]

$$
\forall i=1 \ldots n, \quad \sum_{j \neq i} \frac{\gamma_{i j}}{\left|\vec{r}_{i}-\vec{r}_{j}\right|}=c_{i}
$$

Here, the index $i$ runs over all $n=\sum_{a} N_{a}$ pairs $(a, s)$ with $s=1 \ldots N_{a}$, and the corresponding $\gamma_{i j}$ and $c_{i}$ are equal to $\alpha_{a b}$ and $\zeta_{a}$, in such a way that $\sum_{i=1}^{n} c_{i}=\sum_{a=1}^{K} N_{a} \zeta_{a}=0$. The same equations (2.3) govern the positions of $n$ centers with charges $\gamma_{i}$ such that $\left\langle\gamma_{i}, \gamma_{j}\right\rangle=\gamma_{i j}$ in $\mathcal{N}=2$ supergravity $[1,2]$. The space of solutions modulo common translations is a phase space $\mathcal{M}_{n}\left(\left\{\gamma_{i j}, c_{i}\right\}\right)$ of dimension $2 n-2$, equipped with a natural symplectic form (inherited from the symplectic form on the full non-BPS phase space) such that the moment map for spatial rotations is given by the total angular momentum $\vec{J}=\frac{1}{2} \sum_{i<j} \gamma_{i j}\left(\vec{r}_{i}-\vec{r}_{j}\right) /\left|\vec{r}_{i}-\vec{r}_{j}\right|[47]$. BPS states on the Coulomb branch are harmonic spinors for the natural Dirac operator on $\mathcal{M}_{n}\left(\left\{\gamma_{i j}, c_{i}\right\}\right)[9,47]$.

The Witten index $\Omega(\gamma, z, y)$ defined in (1.1) counts BPS states on the Higgs branch. In the case where the dimension vector $\gamma$ is primitive and the superpotential $\mathcal{W}$ is generic, $\mathcal{M}_{Q}$ is compact, so $\Omega(\gamma, z, y)$ is a symmetric Laurent polynomial in $y$, which can be viewed as the character of the Lefschetz action of $\mathrm{SO}(3)$ on the cohomology of the quiver moduli space $\mathcal{M}_{Q}$. When $\gamma$ is not primitive, $\mathcal{M}_{Q}$ is no longer compact, but one can still define the $\chi$-genus using intersection cohomology. It is useful to introduce the rational invariant [36]

$$
\left.\left.\bar{\Omega}(\gamma, z, y):=\bar{\chi}_{Q}\left(\left\{N_{a}, \zeta_{a}\right)\right\}, y\right):=\sum_{d \mid N_{a}} \frac{y-1 / y)}{d\left(y^{d}-y^{-d}\right)} \chi_{Q}\left(\left\{N_{a} / d, \zeta_{a}\right)\right\}, y^{d}\right),
$$

which coincides with $\left.\chi_{Q}\left(\left\{N_{a}, \zeta_{a}\right)\right\}, y\right)$ when the dimension vector $\gamma=\left(N_{1}, \ldots, N_{K}\right)$ is primitive, but is in general a rational function of $y$ whenever $\gamma$ is not primitive. The advantage is that $\left.\bar{\chi}_{Q}\left(\left\{N_{a}, \zeta_{a}\right)\right\}, y\right)$ satisfies a much simpler wall-crossing formula than $\left.\chi_{Q}\left(\left\{N_{a}, \zeta_{a}\right)\right\}, y\right)[20,21,24]$.

\subsection{The Coulomb branch formula}

The Coulomb branch formula conjecturally expresses the rational index $\bar{\Omega}(\gamma, z, y)$ in terms of single-centered indices $\bar{\Omega}_{S}\left(\gamma_{i}\right)$ as follows [36, 37, 41]:

$$
\left.\bar{\chi}_{Q}\left(\left\{N_{a}, \zeta_{a}\right)\right\}, y\right)=\sum_{\gamma=\sum_{i=1}^{n} \gamma_{i}} \frac{g_{\mathrm{C}}\left(\left\{\gamma_{i}, c_{i}\right\}, y\right)}{\left|\operatorname{Aut}\left\{\gamma_{i}\right\}\right|} \prod_{i=1}^{n} \bar{\Omega}_{\mathrm{T}}\left(\gamma_{i}, y\right)
$$

where $\bar{\Omega}_{\mathrm{T}}\left(\gamma_{i}, y\right)$ is constructed in terms of $\Omega_{\mathrm{T}}\left(\gamma_{i}, y\right)$ by a relation similar to (2.4). The 'total' invariant $\Omega_{\mathrm{T}}\left(\gamma_{i}, y\right)$ is in turn determined in terms of the single-centered invariants $\Omega_{S}\left(\gamma_{i}, y\right)$ via

$$
\Omega_{\mathrm{T}}(\gamma, y)=\Omega_{S}(\gamma, y)+\sum_{\gamma=\sum_{i=1}^{m} m_{i} \beta_{i}} H\left(\left\{\beta_{i}, m_{i}\right\}, y\right) \prod_{i=1}^{m} \Omega_{S}\left(\beta_{i}, y^{m_{i}}\right) .
$$

In (2.5), the sum runs over unordered decompositions of $\gamma$ into a sum of vectors $\gamma_{i}=$ $\sum_{a} n_{i, a} \alpha_{a}$ which are linear combinations of the basis vectors $\alpha_{a}$ with positive integer coefficients. Similarly, in (2.6) the sums run over unordered decompositions of $\gamma$ into sums of 
vectors $m_{i} \beta_{i}$ with $m_{i} \geq 1$ and $\beta_{i}$ a linear combination of the $\alpha_{a}$ 's with positive integer coefficients. ${ }^{3}$ The functions $H\left(\left\{\beta_{i}, m_{i}\right\}, y\right)$ are determined recursively by the so called "minimal modification hypothesis" (see $[37,41]$ for details) and their role is to ensure that the full refined index $\bar{\Omega}(\gamma, z)$ is a symmetric Laurent polynomial in $y$. The function $g_{\mathrm{C}}\left(\left\{\gamma_{i}, c_{i}\right\}, y\right)$, known as the Coulomb index, is the only quantity on the r.h.s. of (2.5) which depends on the stability parameters $\zeta_{a}$. It is defined as the equivariant index of the Dirac operator on the phase space $\mathcal{M}_{n}\left(\left\{\gamma_{i}, c_{i}\right\}\right)$, computed by localization with respect to rotations around a fixed axis $[24,36,37]$. The fixed points of the action of $J_{3}$ on $\mathcal{M}_{n}\left(\left\{\gamma_{i}, c_{i}\right\}\right)$ are collinear black hole solutions, which are classified by permutations $\sigma$ of $\{1,2, \ldots n\}$,

$$
g_{\mathrm{C}}\left(\left\{\gamma_{i}, c_{i}\right\}, y\right)=\frac{(-1)^{n-1+\sum_{i<j} \gamma_{i j}}}{\left(y-y^{-1}\right)^{n-1}} \sum_{\sigma \in S_{n}} F_{C, n}\left(\left\{\gamma_{\sigma(i)}, c_{\sigma(i)}\right\}\right) y^{\sum_{i<j} \gamma_{\sigma(i) \sigma(j)}}
$$

where the 'partial Coulomb index' $F_{C, n}\left(\left\{\gamma_{i} c_{i}\right\}\right) \in \mathbb{Z}$ counts (with sign) collinear solutions for a fixed ordering $x_{1}<x_{2} \cdots<x_{n}$ along the axis. A recursive procedure for computing it was given in [37] and has been implemented in a Mathematica package (see section A).

When the Abelian quiver $Q\left(\left\{\gamma_{i}\right\}\right)$ constructed from the adjacency matrix $\gamma_{i j}=$ $\left\langle\gamma_{i}, \gamma_{j}\right\rangle=\sum_{a, b} n_{i, a} n_{j, b} \kappa_{a b}$ has no oriented loop, the Coulomb index (2.7) coincides with the $\chi$-index $\chi_{Q\left(\left\{\gamma_{i}\right\}\right)}$ with stability parameters $c_{i}=\sum_{a} n_{i, a} \zeta_{a}$, in particular it is a symmetric Laurent polynomial in $y$. When $Q\left(\left\{\gamma_{i}\right\}\right.$ has oriented closed loops, this relation is lost, and the Coulomb index (2.7) is in general a rational function. The functions $H\left(\left\{\beta_{i}, m_{i}\right\}, y\right)$ are then adjusted in such a way that the full index $\bar{\Omega}(\gamma, z)$ obtained via $(2.5)$ is a symmetric Laurent polynomial in $y$, provided the single-centered indices $\Omega_{S}\left(\gamma_{i}, y\right)$ are. The minimal modification hypothesis of [37] gives a unique prescription for computing $H$, based on the assumption that the missing contributions from the boundary of $\mathcal{M}_{n}$ carry the minimal possible angular momentum. Note that this prescription does not take into account the condition of absence of closed timelike curves, which is irrelevant in the context of quiver quantum mechanics, but needs to be checked by hand for general supergravity bound states (see e.g. [36, §3.2] for an example where this condition makes an important difference).

In the special case where the dimension vector $\gamma$ is primitive and such that all charge vectors $\gamma_{i}$ appearing in each decomposition $\gamma=\sum \gamma_{i}$ are distinct and primitive (which in particular applies when the original quiver $Q$ is Abelian), the Coulomb branch formula (2.5) simplifies to

$$
\Omega(\gamma, z, y)=\sum_{\gamma=\sum_{i=1}^{n} \gamma_{i}} g_{\mathrm{C}}\left(\left\{\gamma_{i}, c_{i}\right\}, y\right) \prod_{i=1}^{n}\left\{\Omega_{S}\left(\gamma_{i}, y\right)+\sum_{\sum_{j=1}^{m_{i}} \beta_{j}=\gamma_{i}} H_{m_{i}}\left(\left\{\beta_{j}\right\}, y\right) \prod_{j=1}^{m_{i}} \Omega_{\mathrm{S}}\left(\beta_{j}, y\right)\right\} .
$$

In this case, the rational functions $H\left(\left\{\beta_{j}\right\}, y\right)$ are fixed by demanding that the coefficient of the monomial $\prod_{j=1}^{m} \Omega_{\mathrm{S}}\left(\beta_{j}, y\right)$ in $\Omega(\gamma, z, y)$ be a Laurent polynomial in $y$. Requiring that $H\left(\left\{\beta_{j}\right\}, y\right)$ are invariant under $y \rightarrow 1 / y$ and vanish at $y=\infty$ fixes them uniquely [36]. In [17] it is conjectured that the Hodge polynomial (1.2) of $\mathcal{M}_{Q}$ can be obtained from the

\footnotetext{
${ }^{3}$ If one of the constituents $\beta_{i}$ is not primitive, all choices $\left(d m_{i}, \beta_{i} / d\right)$ are counted as distinct contributions.
} 
$\chi$-genus given by (2.5) or (2.8) by replacing the argument $y$ in $\Omega_{S}\left(\gamma_{i}, y\right)$ by the fugacity $t$ conjugate to $p-q$.

\subsection{The MPS formula}

For quiver without oriented loops, the single-centered invariants $\bar{\Omega}_{S}\left(\gamma_{i}\right)$ vanish unless $\gamma_{i}$ is a multiple of a basis vector $\alpha_{a}$, in which case $\bar{\Omega}_{\mathrm{S}}\left(\ell \alpha_{a}\right)=(y-1 / y) /\left[\ell\left(y^{\ell}-y^{-\ell}\right)\right]$. The Coulomb branch formula therefore reduces to

$$
\bar{\chi}_{Q}\left(\left\{N_{a}, \zeta_{a}\right\}, y\right)=\sum_{\substack{N_{a}=\sum \ell \ell_{\ell} \\ a=1 \ldots K}} \chi_{Q\left(\left\{n_{\ell a}\right\}\right)}\left(\left\{c_{i}\right\}, y\right) \prod_{a=1}^{K} \prod_{\ell} \frac{1}{n_{\ell, a} !}\left[\frac{y-1 / y}{\ell\left(y^{\ell}-y^{-\ell}\right)}\right]^{n_{\ell, a}}
$$

The factor $\chi_{Q\left(\left\{n_{i \ell, a}\right)\right.}\left(\left\{c_{i}\right\}, y\right)$ is the $\chi$-genus of an Abelian quiver with $n=\sum_{\ell, a} n_{\ell, a}$ vertices $\left\{W_{a, \ell, m}, 1 \leq a \leq K, 1 \leq m \leq n_{\ell, a}\right\}$, which we denote by $Q\left(\left\{n_{\ell a}\right\}\right)$ (see figure 1). Denoting the nodes of the 'blown up quiver' by $\left\{W_{i}, 1 \leq i \leq n\right\}$ using an arbitrary bijection $i \mapsto$ $(a(i), \ell(i), m(i))$, the adjacency matrix of $Q\left(\left\{n_{\ell a}\right\}\right)$ is given by $\gamma_{i j}=\ell(i) \ell(j) \kappa_{a(i) a(j)}$, the dimension vector has entries $N_{i}=1$ while the stability vector is $c_{i}=\ell(i) \zeta_{a(i)}$, in such a way that $\sum_{i=1}^{n} c_{i}=\sum_{\ell, a} \ell n_{\ell, a} \zeta_{a}=0$. The positive integer $\ell(i)$ is sometimes called the level of the vertex $W_{i}$. Physically, the quiver $Q\left(\left\{n_{\ell a}\right\}\right)$ describes the interactions of $n=\sum_{\ell} n_{\ell a}$ black holes with charges $\gamma_{i}=\ell(i) \alpha_{a(i)}$, treated as distinguishable particles, so we use the notation the equivalent notation $Q\left(\left\{\gamma_{i}\right\}\right)$ for the quiver $Q\left(\left\{n_{\ell a}\right\}\right)$.

The formula (2.9) is a special case $S=\{1, \ldots, K\}$ of a more general relation operating only on a subset $S \subset\{1, \ldots, K\}$ of the nodes:

$$
\bar{\chi}_{Q}\left(\left\{N_{a}, \zeta_{a}\right\}, y\right)=\sum_{N_{a}=\sum_{\ell \in S} \ell_{\ell, a}} \chi_{Q\left(\left\{n_{\ell, a}\right)\right\}}\left(\left\{c_{i}\right\}, y\right) \prod_{a \in S} \prod_{\ell} \frac{1}{n_{\ell, a} !}\left[\frac{y-1 / y}{\ell\left(y^{\ell}-y^{-\ell}\right)}\right]^{n_{\ell, a}}
$$

where the quiver $Q\left(\left\{n_{\ell, a}\right\}\right)$ is obtained from $Q$ by replacing every vertex $V_{a} \in \mathcal{V}_{Q} \cap S$ with vertices $W_{a, \ell, m}, 1 \leq m \leq n_{\ell, a}$, carrying level $\ell$ while the vertices $V_{a} \in V_{Q} \backslash S$ are unaffected. Again, we denote by $\left\{W_{i}\right\}$ the set of all vertices, and by $a(i), \ell(i), N(i)$ their type, level and dimension, equal to $(a, \ell, 1)$ for $W_{a, \ell, m}$ and to $\left(a, 1, N_{a}\right)$ for $V_{a} \in V_{Q} \backslash S$. The adjacency matrix is given as before by $\gamma_{i j}=\ell(i) \ell(j) \kappa_{a(i) a(j)}$, the stability vector is $c_{i}=\ell(i) \zeta_{a(i)}$ and the dimension vector has entries $N_{i}=N(i)$, in such a way that $\sum_{i=1}^{n} N_{i} c_{i}=0$. Clearly, this relation is only non-trivial if the entries in $S$ correspond to non-Abelian nodes, i.e. $N_{a}>1$ for $a \in S$. Moreover, the relation (2.10) follows iteratively from the special case where $S$ contains a single element. In the case where the dimension vector is primitive, this relation was established in [24, appendix D] based on Reineke's formula [25] for quivers without oriented loops and with primitive dimension vector. It is worth noting that the proof also works for quivers with oriented loops but with vanishing superpotential, since Reineke's formula also holds in that case [17].

\section{Indices from residues}

Using supersymmetric localization techniques, the authors of [31, 33] have found a general prescription for computing the Witten index in any gauged quantum mechanics with 


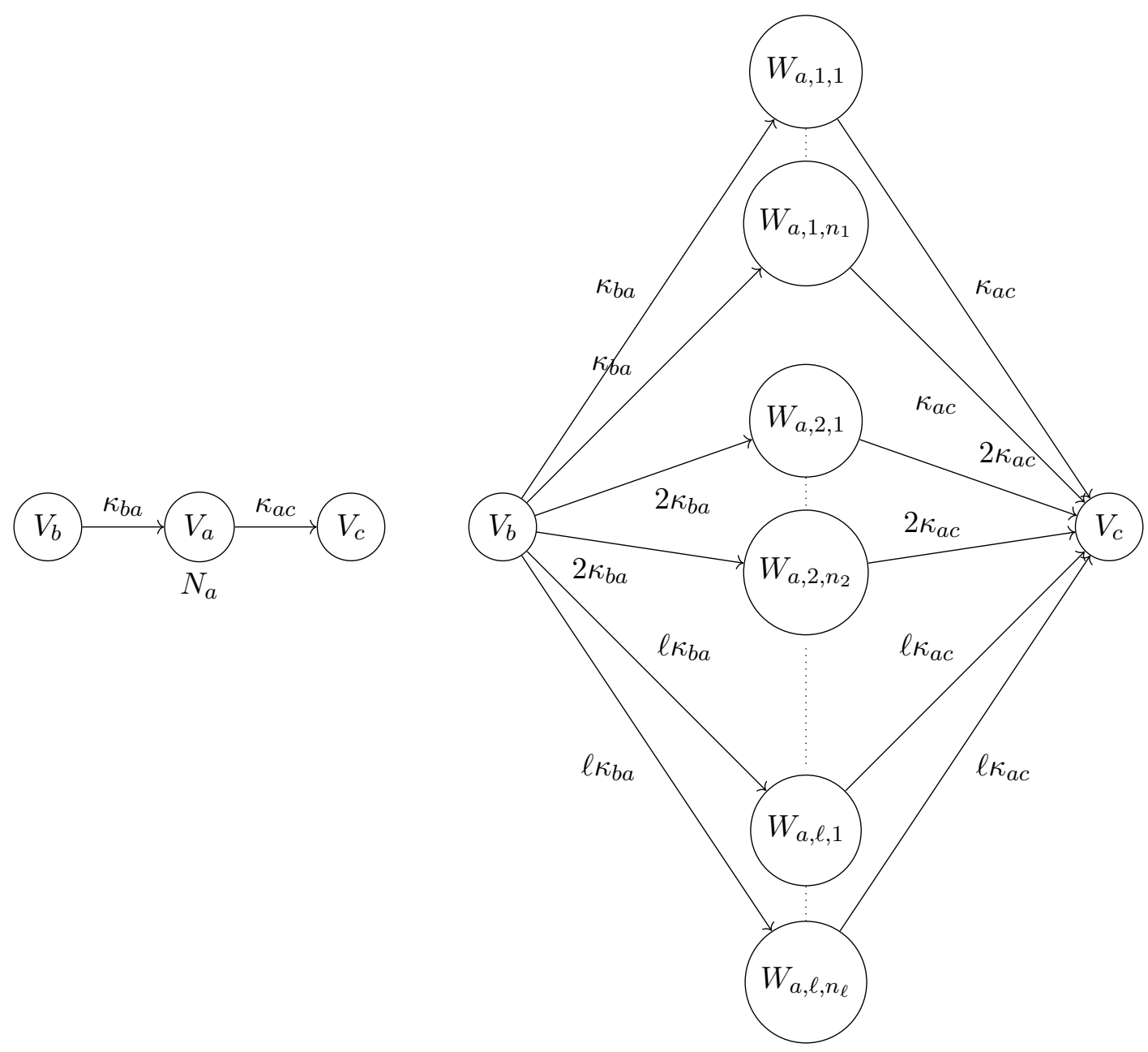

Figure 1. Blowing up a non-Abelian node of rank $N_{a}=\sum_{\ell} \ell n_{a, \ell}$ (on the left) into $\sum_{\ell} n_{a, \ell}$ Abelian nodes (on the right). The number of arrows from $W_{a, \ell, m}$ to $V_{b}$ is $\ell$ times the number of arrows from $V_{a}$ to $V_{b}$, where $\ell$ is the level of the node $W_{a, \ell, m}$. This operation may be performed on any number of non-Abelian nodes.

two supercharges. In the case of quiver quantum mechanics with four supercharges, the prescription reads:

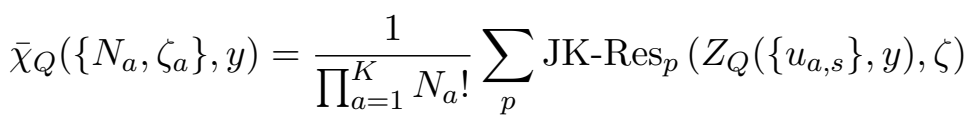

Here, the variables $u_{a, s}$ parametrize the complexified Cartan torus $\prod_{a=1}^{K}\left(\mathbb{C}^{\times}\right)^{N_{a}}$ of the gauge group $\left[\prod_{a} \mathrm{U}\left(N_{a}\right)\right] / \mathrm{U}(1)$, the factor $Z_{Q}\left(\left\{u_{a, s}\right\}, y\right)$ is a holomorphic top form on this space and the sum runs over all poles of $Z_{Q}\left(\left\{u_{a, s}\right\}, y\right)$. The symbol JK-Res denotes a specific prescription, originally due to Jeffrey and Kirwan [34], ${ }^{4}$ for extracting the residue at the corresponding pole. The prescription crucially depends on the stability vector $\zeta$, and ensures that the result is consistent with the wall-crossing formula. The function

\footnotetext{
${ }^{4}$ The specific prescription used in [28, 31] and in this paper can be found in [35].
} 
$Z_{Q}\left(\left\{u_{a, s}\right\}, y\right)$ originates from the one-loop fluctuation determinant of all fields in the vector and chiral multiplets, and is given by

$$
\begin{aligned}
& Z_{Q}\left(\left\{u_{a, s}\right\}, y\right)=\left[\frac{\pi \hbar}{\sin (\pi \hbar)}\right]^{\sum_{a=1}^{K} N_{a}-1} \prod_{\substack{a=1 \ldots K \\
s, s^{\prime}=1 \ldots, N_{a} \\
s \neq s^{\prime}}} \frac{\sin \left[\pi \hbar\left(u_{a, s^{\prime}}-u_{a, s}\right)\right]}{\sin \left[\pi \hbar\left(u_{a, s}-u_{a, s^{\prime}}-1\right)\right.} \\
& \times \prod_{\substack{a, j=1 \ldots K \\
\kappa_{a b}>0}} \prod_{\substack{s=1 \ldots N_{a} \\
s^{\prime}=1 \ldots N_{b}}} \prod_{A=1 \ldots \kappa_{a b}} \frac{\sin \left[\pi \hbar\left(u_{b, s^{\prime}}-u_{a, s}+1-\frac{R_{a b}}{2}-\theta_{a b, A}\right)\right]}{\sin \left[\pi \hbar\left(u_{a, s}-u_{b, s^{\prime}}+\frac{R_{a b}}{2}+\theta_{a b, A}\right)\right]} \\
& \times \prod_{\substack{a=1 \ldots K \\
s=1 \ldots N_{a}}} \mathrm{~d} u_{a, s}
\end{aligned}
$$

where $y=e^{i \pi \hbar}$, and $\widetilde{\prod}$ denotes the omission of any one of the $\mathrm{d} u_{a, s}$ 's in the measure (due the decoupling of the diagonal U(1) action). The quantity $R_{a b}$ denotes the $R$-charge of the chiral fields $\phi_{a b, A, s s^{\prime}}$, while the parameters $\theta_{a b, A}$ are chemical potentials ${ }^{5}$ for the $\mathrm{U}\left(\kappa_{a b}\right)$ flavor symmetry permuting the chiral fields $\phi_{a b, A, s s^{\prime}}$ with $A=1 \ldots \kappa_{a b}$. In the absence of oriented loops, the assignment of $R$-charges is irrelevant, but if oriented loops are present, it constrains the possible superpotential, which must be a gauge invariant polynomial in the $\phi_{a b, A, s s^{\prime}}$ with R-charge equal to 2, invariant under flavor symmetries with non-zero potential $\theta_{a b, A}$. In particular, in order to allow for a generic superpotential, one should set all flavor potentials to zero and ensure that the total R-charge for any oriented loop is equal to 2 . Clearly, (3.2) is invariant under $\hbar \rightarrow-\hbar$, or equivalently $y \rightarrow 1 / y$.

In the limit $y \rightarrow 1$ (or $\hbar \rightarrow 0$ ), and for vanishing chemical potentials $\theta_{a b, A}=0$, the $\chi$-genus reduces to the Euler number, given by the sum of residues of

$$
\begin{aligned}
Z_{Q}\left(\left\{u_{a, s}\right\}\right)= & \prod_{\substack{a=1 \ldots K \\
s, s^{\prime}=1 \ldots, N_{a} \\
s \neq s^{\prime}}} \frac{u_{a, s^{\prime}}-u_{a, s}}{u_{a, s}-u_{a, s^{\prime}}-1} \prod_{\substack{a, b=1 \ldots K \\
\kappa_{a b}>0}} \prod_{\substack{s=1 \ldots N_{a} \\
s^{\prime}=1 \ldots N_{b}}}\left[\frac{u_{b, s^{\prime}}-u_{a, s}+1-\frac{R_{a b}}{2}}{u_{a, s}-u_{b, s^{\prime}}+\frac{R_{a b}}{2}}\right]^{\kappa_{a b}} \\
& \times \prod_{\substack{a=1 \ldots K \\
s=1 \ldots N_{a}}} \mathrm{~d} u_{a, s}
\end{aligned}
$$

When $\theta_{a b, A} \neq 0$, the limit $\hbar \rightarrow 0$ instead produces the equivariant Euler number for the action of the Cartan torus of the flavor symmetry. It is worth noting that (3.2) arises as the limit $\tau \rightarrow \mathrm{i} \infty$ from the elliptic genus of a two-dimensional gauged linear sigma model with the same matter content, given by a sum of residues of

$$
\begin{aligned}
& Z_{Q}\left(\left\{u_{a, s}\right\}, y, \tau\right)=\left[\frac{2 \pi \eta^{3} \hbar}{\theta_{1}(\hbar)}\right]^{\sum_{a=1}^{K} N_{a}-1} \prod_{\substack{a=1 \ldots K \\
s, s^{\prime}=1 \ldots N_{a} \\
\text { sfs' } \\
\vartheta_{j}}} \frac{\vartheta_{1}\left[\pi \hbar\left(u_{a, s^{\prime}}-u_{a, s}\right)\right]}{\vartheta_{1}\left[\pi \hbar\left(u_{a, s}-u_{a, s^{\prime}}-1\right)\right.} \\
& \times \prod_{\substack{a, j=1 \ldots K \\
\kappa_{a b}>0}} \prod_{\substack{s=1 \ldots N_{a} \\
s^{\prime}=1 \ldots N_{b}}}\left[\frac{\vartheta_{1}\left[\pi \hbar\left(u_{b, s^{\prime}}-u_{a, s}+1-\frac{R_{a b}}{2}\right)\right]}{\vartheta_{1}\left[\pi \hbar\left(u_{a, s}-u_{b, s^{\prime}}+\frac{R_{a b}}{2}\right)\right]}\right]^{\kappa_{a b}} \prod_{\substack{a=1 \ldots K \\
s=1 \ldots N_{a}}} \mathrm{~d} u_{a, s}
\end{aligned}
$$

\footnotetext{
${ }^{5}$ Since the R-charge is defined only up to the addition of flavor symmetries, one may decide to absorb $\theta_{a b, A}$ into $R_{a b, A}=R_{a b}+2 \theta_{a b, A}$. However we find it convenient to separate $R_{a b, A}$ into a flavor invariant part $R_{a b}$ and chemical potentials $\theta_{a b, A}$ which may break part of the flavor symmetry.
} 
where $\theta_{1}(v, \tau)=\theta_{1}(v)=2 q^{1 / 8} \sin (\pi v) \prod_{n=1}^{\infty}\left(1-q^{n}\right)\left(1-q^{n} e^{2 \pi \mathrm{i} v}\right)\left(1-q^{n} e^{-2 \pi \mathrm{i} v}\right)$. Note however that in $0+1$ dimensions, there is no need to cancel the R-symmetry anomaly, unlike for the elliptic genus of two-dimensional sigma models.

In order to compute the residue in (3.1), it is more efficient to change variables to $v_{a, s}=e^{2 \pi \mathrm{i} \hbar u_{a, s}}$, such that (3.2) may be replaced by

$$
\begin{aligned}
Z_{Q}\left(\left\{v_{a, s}\right\}, y\right)= & \prod_{\substack{a=1 \ldots K \\
s, s^{\prime}=\ldots \ldots N_{a} \\
s \neq s^{\prime}}} \frac{v_{a, s^{\prime}}-v_{a, s}}{v_{i, s} / y-y v_{a, s^{\prime}}} \\
& \times \prod_{\substack{a, b=1 \ldots K \\
\kappa_{a b}>0}} \prod_{\substack{s=1 \ldots N_{a} \\
s^{\prime}=1 \ldots N_{b}}} \prod_{A=1 \ldots \kappa_{a b}} \frac{y^{1-R_{a b}} \nu_{a b, A} v_{b, s^{\prime}}-v_{a, s} / y}{v_{a, s}-y^{-R_{a b}} \nu_{a b, A} v_{b, s^{\prime}}} \prod_{\substack{a=1 \ldots K \\
s=1 \ldots N_{a}}} \frac{\mathrm{d} v_{a, s}}{v_{a, s}(y-1 / y)}
\end{aligned}
$$

where $\nu_{a b, A}=e^{-\mathrm{i} \pi \theta_{a b, A}}$ are flavor fugacities. In this representation, the symmetry under $y \rightarrow 1 / y$ requires inverting $v_{a, s} \rightarrow 1 / v_{a, s}, \alpha_{a b, A} \rightarrow 1 / \alpha_{a b, A}$.

\subsection{Review of the JK residue prescription}

To explain the prescription for identifying and extracting the relevant residues, let us introduce the weight lattice $\Lambda=\mathbb{Z}^{\sum N_{a}}$, with basis $e_{a, s}, a=1 \ldots K, s=1 \ldots N_{a}$. To each root of $\mathrm{U}\left(N_{a}\right)$ we associate the vector $e_{a, s}-e_{a, s^{\prime}}$ dual to the hyperplane $H_{a, s, s^{\prime}}=$ $u_{a, s}-u_{a, s^{\prime}}-1$. To each bifundamental chiral field $\phi_{a b, A, s s^{\prime}}$ with $\kappa_{a b}>0$ we associate the vector $e_{a, s}-e_{b, s^{\prime}}$ dual to the hyperplane $H_{a b, A, s s^{\prime}}=u_{a, s}-u_{b, s^{\prime}}+\frac{R_{a b}}{2}+\theta_{a b, A}$. If the flavor chemical potentials $\theta_{a b, A}$ vanish for all $A$, we may as well consider a single hyperplane $H_{a b, s s^{\prime}}$ with multiplicity $\left|\kappa_{a b}\right|$. Finally, since all fields are neutral under the diagonal U(1) action, we set one Cartan parameter to zero, e.g. $u_{1,1}=0$, and disregard the corresponding entry of all charge vectors. We denote by $r=\sum_{a=1}^{K} N_{a}-1$ the rank of the reduced lattice $\Lambda / \mathbb{Z}$.

Now, for each isolated intersection $p$ of hyperplanes, let $\mathcal{H}_{p}$ be the list of hyperplanes intersecting at $p$; to construct this list, consider all $r$-plets of hyperplanes, determine their intersection, ${ }^{6}$ collect all non-degenerate intersection points, and finally for each point in this list, collect all hyperplanes which meet at that point. Let $\mathcal{Q}_{p}$ be the corresponding list of charge vectors. We further assume that the intersection is projective, i.e. that all vectors in $\mathcal{Q}_{p}$ lies in a positive half-space of $\mathbb{R}^{r}$. If that is not the case, it is usually possible to perturb the charges $R_{a b}$ so as to resolve a non-projective intersection into multiple projective intersections.

Let $\mathcal{F}_{p}$ be the list of flags $F=\left(F_{1}, \ldots, F_{r}\right)$ made out of vectors in $\mathcal{Q}_{p}$; equivalently, the list of ordered $r$-plets $Q_{F}=\left(Q_{1}, \ldots Q_{r}\right)$ of vectors in $\mathcal{Q}_{p}$, subject to the equivalence relation $Q_{F} \sim Q_{F}^{\prime}$ if $Q_{F} Q_{F}^{\prime-1}$ is a lower triangular matrix, if the rows of $Q_{F}$ denote the charge vectors. The space spanned by $Q_{j}$ with $1 \leq i \leq j$ defines the $i$-th graded space $F_{i}$

\footnotetext{
${ }^{6}$ For quiver theories, each non-degenerate $r$-plet of hyperplanes appears to have only one intersection point on the Cartan torus, but this is not true for more general matter content.
} 
in the flag $F$. Let $\kappa_{F, i}$ be the sum of all charge vectors ${ }^{7}$ in $\mathcal{Q}_{p}$ which belongs to $F_{i}$, for all $i=1, \ldots r$. Note that $\kappa_{F, i}$ includes contributions from the vectors $Q_{1}, \ldots, Q_{i}$, but it may also include charge vectors which do not belong to the list $\left(Q_{1}, \ldots Q_{r}\right)$ if more than $r$ hyperplanes intersect at $p$, i.e. if the intersection is degenerate. In particular, $\kappa_{F, r}$ is the sum of all vectors in $\mathcal{Q}_{p}$, irrespective of the flag $F$. Note also that the matrix $\kappa_{F}$ is independent of the choice of representative $Q_{F}$ for the flag $F$.

Now, let us promote the stability condition $\zeta \in \mathbb{R}^{K}$ to a vector $\eta \in \mathbb{R}^{r}$, by using the diagonal embedding $\eta_{a, s}=\zeta_{a}$, and perturbing slightly away from this point (the perturbation must be chosen once for all, and be the same for all flags). A flag $F \in \mathcal{F}_{p}$ is said to be stable if $\eta$ belongs to the positive cone spanned by the vectors $\kappa_{F, i}$ with $i=1 \ldots r$, i.e.

$$
\eta=\sum_{i=1}^{r} \lambda_{i} \kappa_{F, i}, \quad \lambda_{i}>0
$$

In the case where the vectors $\kappa_{i}(F)$ are linearly dependent, we discard the corresponding flag, since they will not be stable for generic values of $\eta$. Let $\mathcal{F}_{p}(\eta)$ be the set of stable flags constructed from the list of hyperplanes $\mathcal{H}_{p}$ meeting at $p$. The Jeffrey-Kirwan residue at $p$ is then the sum of iterated residues

$$
\operatorname{JK}_{-\operatorname{Res}_{p}}(Z(u), \eta)=\sum_{F \in \mathcal{F}_{p}(\eta)} \operatorname{sign}(\operatorname{det} \kappa) \operatorname{Res}_{\tilde{u}_{r}=0} \ldots \operatorname{Res}_{\tilde{u}_{1}=0} \tilde{Z}(\tilde{u})
$$

where $\tilde{u}_{i}=H_{Q_{i}}(u)$ (or in matrix notation, $\tilde{u}=u \cdot Q_{F}$ ) and $\tilde{Z}(\tilde{u})=Z(u) \mathrm{d} \tilde{u} / \mathrm{d} u$. The residue is most efficiently computed from the representation (3.5), but one must of course keep track of the Jacobians $\partial v / \partial u$ and $\partial u / \partial \tilde{u}$.

It is worth stressing that while the condition (3.6) restricts the possible flags contributing to the index, it frequently happens that some of the allowed flags give a vanishing residue. In particular, note that in the absence of flavor fugacities and upon treating the hyperplanes $H_{a b, A, s s^{\prime}}, A=1 \ldots \kappa_{a b}$ as a single hyperplane of degree $\kappa_{a b}$, the stability condition for flags depends only on the sign of $\kappa_{a b}$, not on its absolute value, but the complex dimension $d$ of the moduli space $] \mathcal{M}_{Q}$ does depend on $\left|\kappa_{a b}\right|$, and the $\chi$-genus must vanish if $d<0$. It would be interesting to find a criterium predicting when a given stable flag will produce a vanishing contribution.

\subsection{Residues and fixed points}

As explained in [31, 32], the residue formula (3.1) arises by applying supersymmetric localization to the functional integral defining the gauge theory on an Euclidean circle of radius $\beta$. For this, one constructs a linear combination $Q_{\hbar}$ of the 4 supercharges which squares to a combination $Q_{\hbar}^{2}=J$ of a $\mathrm{U}(1)_{R}$ rotation and global flavor symmetries, such that the action becomes $Q_{\hbar}$-exact. Fixed points of $Q_{\hbar}$ are such that the complexified scalars $u_{a, s}$ in the Cartan part of vector multiplets become constant (independent of the coordinate $\tau$ along the thermal circle), while the chiral multiplets must vanish for generic values of the

\footnotetext{
${ }^{7}$ Here it matters whether we treat hyperplanes with $\left|\kappa_{a b}\right|>1$ as multiple copies with unit multiplicity, or a single copy with multiplicity $\left|\kappa_{a b}\right|$. We find in examples that the two prescriptions lead to the same result.
} 
$u_{a, s}$ 's. The one-loop determinant for the off-diagonal components of the vector multiplets and for the chiral multiplets gives a $(r, r)$ form $\omega$ on the space of the $u_{a, s}$ 's, which has the topology of a cylinder $\left(\mathbb{C}^{\times}\right)^{r}=\left(\mathbb{R} \times S_{1}\right)^{r}$. By integration by parts, the integral can be rewritten as a sum of contour integrals around the poles at finite and infinite distance. At poles at finite distance in the $u$-plane, corresponding to an intersection of hyperplanes $H_{1}, \ldots H_{r}$, the chiral multiplets (in case $H_{i}=H_{a b, A, s s^{\prime}}$ ) or the off-diagonal scalars in the vector multiplets (in case $H_{i}=H_{a, s, s^{\prime}}$ ) may acquire a non-zero vev. These correspond to fixed points of the one-parameter subgroup of the flavor symmetry $\prod_{a, b, \kappa_{a b}>0} \mathrm{U}\left(\kappa_{a b}\right)$ acting on the Higgs branch. The condition (3.6) ensures that fixed points with $\phi_{a b, A, s s^{\prime}} \neq 0$ for $a, b, s, s^{\prime}$ associated to the hyperplanes $H_{i}$ are allowed by the D-term constraints (2.1). Moreover, the contribution of each fixed point agrees with the Atiyah-Bott Lefschetz fixed point theorem [48] for a Kähler manifold $X$ with a holomorphic action of $f: X \rightarrow X$, which lifts to an action $f^{*}$ on its cohomology $H^{*}(X)$,

$$
\sum_{p, q \geq 0}(-1)^{p+q-d} y^{2 p-d} \operatorname{Tr}_{H^{p, q}} f^{*}=\sum_{p \geq 0}(-1)^{p-d} y^{2 p-d} \sum_{f(P)=P} \frac{\operatorname{Tr}\left(\lambda^{p} \mathrm{~d} f_{P}\right)}{\operatorname{det} \mathbb{C}\left(1-\mathrm{d} f_{P}\right)}
$$

where $\mathrm{d} f_{P}$ is the linear action of $f$ on the holomorphic tangent space at the fixed point $P$, and $\lambda^{p} \mathrm{~d} f_{P}$ is the $p$-th antisymmetric power of this map. In our context, $f$ is the $\mathrm{U}(1)$ flavor symmetry determined by the chemical potentials $\theta_{a b, A}$. In section 4 below we discuss the relation between poles and fixed points in more detail in the case of Abelian quivers.

\subsection{Index in attractor chamber}

For any quiver, there is a special choice of stability conditions known as the attractor chamber [49]

$$
\zeta_{a}^{*}=-\sum_{b} \kappa_{a b} N_{b}
$$

This chamber precludes the existence of two-centered bound states, since for any splitting $\gamma=\gamma_{L}+\gamma_{R}$ of the dimension vector, or equivalently $N_{a}=N_{a}^{L}+N_{a}^{R}$, the DSZ product $\left\langle\gamma_{L}, \gamma_{R}\right\rangle=\kappa_{a b} N_{L}^{a} N_{R}^{b}$ and the effective stability parameter $\zeta_{L}=N_{L}^{a} \zeta_{a}^{\star}=-N_{R}^{a} \zeta_{a}^{\star}$ have opposite sign. For a quiver without oriented loops, the index in this chamber automatically vanishes. This is because such a quiver always admits (possibly more than) one sink, where all arrows are incoming, and (possibly more than) one source, where all arrows are outgoing. The D-term conditions (2.1) have no solutions unless $\zeta_{a}<0$ for a sink, and $\zeta_{a}>0$ for a source. In contrast, at the attractor point $\zeta_{a}^{*}>0$ for sink, and $\zeta_{a}^{*}<0$ for a source. By the same token, the index vanishes at the attractor point for any quiver which admits a sink or source, whether or not it contains an oriented loop; more generally the index vanishes for any stability condition of the form $\zeta_{a}=-\mu_{a} \sum_{b} \kappa_{a b} N_{b}$ such that $\mu_{a}>0$ provided $\sum_{a} N_{a} \zeta_{a}=0$

It is easy to see that the JK residue prescription is consistent with this vanishing property. Indeed, summing up the relation (3.6) over the indices $s=1 \ldots N_{a}$ for each $a$, we get

$$
\zeta=\sum_{i=1}^{r} \lambda_{i} \tilde{\kappa}_{i}
$$


where $\tilde{\kappa}_{i} \in \mathbb{R}^{K}$ gets contributions only from chiral multiplet charge vectors in $\mathcal{Q}_{p}$. If $a$ is a sink, all the contributions to the $a$-th component of $\tilde{\kappa}_{i}$ are strictly negative, so there are no stable flags when $\zeta_{a}>0$, in particular at the attractor point $\zeta^{*}$. Similarly, if $a$ is a source, sink, all the contributions to the $a$-th component of $\tilde{\kappa}_{i}$ are strictly positive, so there are again no stable flags when $\zeta_{a}<0$, in particular at the attractor point $\zeta^{*}$.

In the absence of sources or sinks, in particular for quivers with oriented loops, the index at the attractor point does not necessarily vanishes, but rather gets contributions from single-centered invariants and from scaling solutions thereof, as we discuss further in section 4.3.

\subsection{Cauchy-Bose formula}

One of the bottlenecks in the practical evaluation of computation of (3.1) is the enumeration of intersections points and flags. Each non-Abelian group contributes $\mathcal{O}\left(N_{a}^{2}\right)$ singular hyperplanes, while the rank grows only linearly in $N_{a}$, so the number of possible $\ell$-plets grows exponentially. One remedy is to use the Cauchy-Bose identity (1.3) to rewrite the vector multiplet determinant in (3.2) as

$$
\prod_{\substack{s, s^{\prime}=1 \ldots N \\ s \neq s^{\prime}}} \frac{\sin \left[\pi \hbar\left(u_{s^{\prime}}-u_{s}\right)\right]}{\sin \left[\pi \hbar\left(u_{s}-u_{s^{\prime}}-1\right)\right]}=\sum_{\sigma \in S_{N}} \frac{\epsilon(\sigma) \sin (\pi \hbar)^{N}}{\prod_{s=1}^{N} \sin \left[\hbar\left(u_{s}-u_{\sigma(s)}+1\right)\right]}
$$

Equivalently, in terms of the exponentiated variables $v_{s}=e^{2 \pi \mathrm{i} \hbar u_{s}}$,

$$
\frac{1}{\prod_{s=1}^{N} v_{s}} \prod_{\substack{s, s^{\prime}=1 \ldots . \\ s \neq s^{\prime}}} \frac{\left(v_{s^{\prime}}-v_{s}\right)}{v_{s} / y-y v_{s^{\prime}}}=(1 / y-y)^{N} \sum_{\sigma \in S_{N}} \frac{\epsilon(\sigma)}{\prod_{s=1}^{N}\left(v_{s} / y-y v_{\sigma(s)}\right)}
$$

Using the elliptic generalization of (1.3) due to Frobenius [50]

$$
\operatorname{det} \frac{1}{\theta_{1}\left(\mu_{i}-\nu_{j}\right)}=\frac{\prod_{i<j} \theta_{1}\left(\mu_{i}-\mu_{j}\right) \theta_{1}\left(\nu_{j}-\nu_{i}\right)}{\prod_{i, j} \theta_{1}\left(\mu_{i}-\nu_{j}\right)},
$$

we may similarly write the vector multiplet determinant for the elliptic genus in (3.2) as

$$
\prod_{\substack{s, s^{\prime}=1 \ldots N \\ s \neq s^{\prime}}} \frac{\vartheta_{1}\left[\pi \hbar\left(u_{s^{\prime}}-u_{s}\right)\right]}{\vartheta_{1}\left[\pi \hbar\left(u_{s}-u_{s^{\prime}}-1\right)\right]}=\sum_{\sigma \in S_{N}} \frac{\epsilon(\sigma) \vartheta_{1}(\pi \hbar)^{N}}{\prod_{s=1}^{N} \vartheta_{1}\left[\hbar\left(u_{s}-u_{\sigma(s)}+1\right)\right]}
$$

In each of these formulae, the denominator now involves $N$ hyperplanes rather than $N(N-1) / 2$, which drastically simplifies the classification of intersections flags, though it requires sifting through $N$ ! permutations rather than a single product of many hyperplanes. Fortunately, all permutations in the same conjugacy class (labelled by the partition $\left.\lambda=\sum_{\ell} \ell n_{\ell}\right)$ turn out to produce the same contribution, ${ }^{8}$ so it suffices to pick one particular permutation for each partition, and multiply the result by $\prod_{\ell} \ell^{n_{\ell}} n_{\ell} !$, corresponding

\footnotetext{
${ }^{8}$ This fact is non-trivial since the generalized stability vector $\eta \in \mathbb{R}^{r}$ breaks the $S_{N}$ symmetry. The integrand coming from different permutations with the same cycle shape can be mapped to the same integrand by relabelling the integration variables $u_{s, a}$, at the expense of permuting the entries in the flag $F=\left(Q_{1}, \ldots, Q_{r}\right)$ used for integrating each of them. For non-degenerate intersections, these permutations do not affect the residue, so the result is the same for all permutations with the same cycle shape. For degenerate intersections, the change of variables typically permutes the flags as well (not only the entries $Q_{i}$ in a given flag) and a more detailed analysis is required.
} 
to the number of permutations in the same conjugacy class. As we shall see in section 5 , if one applies this trick for each of the gauge groups $\mathrm{U}\left(N_{a}\right)$, the resulting sum of multipartitions coincides with the MPS formula, at least in the case of non-Abelian quivers without oriented loops.

\section{Abelian quivers}

In this section, we apply the residue formula in the context of Abelian quivers with or without loops. In the absence of oriented loops, we show that the stability condition (3.6) on flags coincides with the condition for existence of fixed points satisfying the D-term equations.

\subsection{Abelian quivers without loops}

To demonstrate this in a simple example, let us focus on Abelian quivers without oriented loops (but possibly with non-oriented ones). The D-term equations (2.1) can be written as

$$
\zeta=\sum_{a, b ; \kappa_{a b}>0}\left|\phi_{a b}\right|^{2}\left(e_{a}-e_{b}\right), \quad\left|\phi_{a b}\right|^{2}:=\sum_{A=1}^{\kappa_{a b}}\left|\phi_{a b, A}\right|^{2}
$$

For a quiver without (oriented nor unoriented) loop, the rank $r=K-1$ is equal to the total number of edges $E=\#\left\{\left(a, b \in \mathcal{A}_{Q}, \kappa_{a b}>0\right\}\right.$ (counted without multiplicity), so each hyperplane $H_{i}$ originates from a set of chiral fields $\phi_{i, A} \equiv \phi_{a(i) b(i), A}$, and vice-versa, and (4.1) becomes

$$
\zeta=\sum_{i=1}^{r}\left|\phi_{i}\right|^{2} Q_{i}
$$

For the flag $F=\left(Q_{1}, \ldots Q_{r}\right)$, one has $\kappa_{F, i}=\sum_{k=1}^{i} Q_{i}$ and it is easy to check that the solution to $\zeta=\sum_{i=1}^{r} \lambda_{i} \kappa_{F, i}$ is given by

$$
\lambda_{i}=\left|\phi_{i}\right|^{2}-\left|\phi_{i+1}\right|^{2} \quad \text { for } \quad i<r, \quad \lambda_{r}=\left|\phi_{r}\right|^{2}
$$

This gives a transparent interpretation of the parameters $\lambda_{i}$ in (3.6) as difference of vevs of chiral fields. In particular, the flag $F$ is stable if the stability parameters allow for solutions of the D-term equations where the only non-vanishing chiral fields $\phi_{i}$ satisfy $\left|\phi_{1}\right|^{2}>\left|\phi_{2}\right|^{2}>\cdots>\left|\phi_{r}\right|^{2}>0$. Different flags correspond to different ordering of these vevs. The associated residue then computes the contribution of the fixed points satisfying these constraints.

As a simple example, consider the Abelian 'star' quiver with $K$ vertices $V_{0}, V_{1}, \ldots V_{K-1}$, with $a_{i}$ arrows from $V_{i}$ to $V_{0}$, with $a_{i}$ a non-zero integer of arbitrary sign. We denote this 
quiver by $Q=S_{a_{1}, \ldots a_{K}}$ :

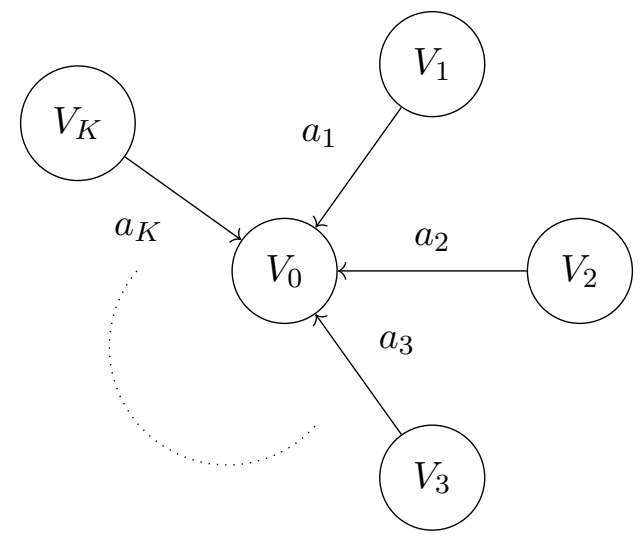

The D-term conditions

$$
\operatorname{sgn}\left(a_{i}\right) \sum_{A=1}^{\left|a_{i}\right|}\left|\phi_{i, A}\right|^{2}=\zeta_{i}, \quad i=1 \ldots K-1
$$

admit solutions only when $\operatorname{sgn}\left(a_{i}\right)=\operatorname{sgn}\left(\zeta_{i}\right)$, in which case the quiver moduli space reduces to a product of projective spaces $\mathcal{M}_{Q}=\prod_{i} \mathbb{P}^{\left|a_{i}\right|-1}$. This agrees with the result from the residue formula

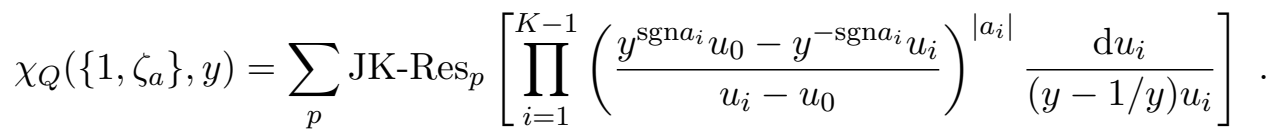

In this case, the singular hyperplanes $u_{i}=u_{0}$ have a non-degenerate intersection and a single stable flag contributes, which depends on the ordering of the $\zeta_{i}$ 's. The result however is independent of that ordering, since the integral factorizes into a product of residues in each variable $u_{i}$ :

$$
\chi_{Q}\left(\left\{1, \zeta_{a}\right\}, y\right)=\prod_{i=1}^{K-1}\left[(-1)^{a_{i}-1} \frac{y^{\left|a_{i}\right|}-y^{-\left|a_{i}\right|}}{y-1 / y}\right]
$$

In the presence of chemical potentials $\theta_{i, A}$ for the global symmetry group $\prod_{i} \mathrm{U}\left(\left|a_{i}\right|\right)$, the intersection at the origin splits into $\prod_{i}\left|a_{i}\right|$ intersection points, corresponding to a choice of $A_{i} \in\left\{1, \ldots,\left|a_{i}\right|\right\}$ for each arrow, producing

$$
\chi_{Q}\left(\left\{1, \zeta_{a}\right\}, y, \theta\right)=\prod_{i=1}^{K-1}\left[\sum_{A_{i}=1}^{\left|a_{i}\right|} \prod_{A \neq A_{i}} \frac{\nu_{i, A} / y-\nu_{i, A_{i}} y}{\nu_{i, A_{i}}-\nu_{i, A}}\right]
$$

Each of these contributions correspond to one fixed point with $\phi_{i, A}=\sqrt{\left|\zeta_{i}\right|} \delta_{A, A_{i}}$, up to $\mathrm{U}(1)$ gauge rotations of the phase of $\phi_{i, A}$. The determinant $\operatorname{det} \mathbb{C}\left(1-\mathrm{d} f_{P}\right)$ appearing in the Lefschetz fixed point formula (3.8) evaluates to $\prod_{i} \prod_{A \neq A_{i}}\left(1-\nu_{i, A} / \nu_{j, A_{i}}\right)$, in agreement with the denominator in (4.7). After summing over $A \neq A_{i}$, each bracket in (4.7) reduces to the corresponding bracket in (4.6), as expected since each factor $\mathbb{P}^{\left|a_{i}\right|-1}$ is compact. Moreover, as $y \rightarrow 1$, each of these non-degenerate intersections contributes \pm 1 , so that $\chi_{Q}\left(\left\{1, \zeta_{a}\right\}, y\right)$ counts the number of fixed points, up to an overall sign. 


\subsection{Abelian quivers with unoriented loops}

For Abelian quivers with $h$ unoriented loops, the rank $r=K-1$ is equal to $E-h$, where $E$ is the total of number of edges (not counting multiplicity). Choosing R-charges $R_{a b}$ and flavor fugacities $\theta_{a b, A}$ such that only non-degenerate intersections of $r$ hyperplanes $\left(Q_{1}, \ldots Q_{r}\right)$ contribute, then only the chiral fields corresponding to $Q_{1}, \ldots Q_{r}$ can get nontrivial expectation values at the intersection, while the remaining $E-r=h$ must be set to zero. Removing these arrows from the original quiver $Q$ defines a 'reduced' quiver $\widetilde{Q}$. In order for the matrix $\kappa_{F}$ to be non-degenerate, it is easy to see that $\widetilde{Q}$ must be a tree, which is therefore a spanning tree of the original quiver $Q$. The same argument as in the previous section shows that the stable flags are again in one-to-one correspondence with fixed points satisfying the D-term equations, for any ordering of the vevs $\left|\phi_{i}\right|^{2}$ along the spanning tree.

\subsubsection{Three node quiver}

As an example with one unoriented loop, let us consider a quiver with three nodes $V_{1}, V_{2}, V_{3}$ and $a=\gamma_{12}<0, b=\gamma_{23}>0, c=\gamma_{31}<0$, which we denote by $Q=C_{a, b, c}$ :

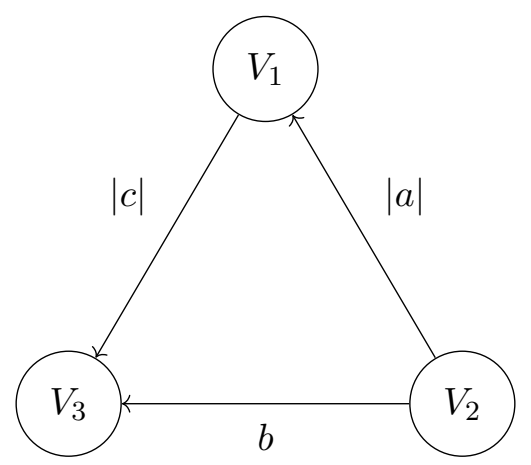

The D-term equations

$$
\left|\phi_{13}\right|^{2}-\left|\phi_{21}\right|^{2}=\zeta_{1}, \quad\left|\phi_{23}\right|^{2}+\left|\phi_{21}\right|^{2}=\zeta_{2}, \quad-\left|\phi_{13}\right|^{2}-\left|\phi_{23}\right|^{2}=\zeta_{3}
$$

admit solutions only when $\zeta_{2}>0$ and $\zeta_{3}<0$. If $\zeta_{1}>0$, then $\phi_{13} \neq 0$ defines a point in $\mathbb{P}^{|c|-1}$, fibered over $\mathbb{P}^{|a|+b-1}$ parametrized by $\left(\phi_{21}, \phi_{23}\right) \neq(0,0)$, with index

$$
\chi_{Q}^{+}(y)=(-1)^{a+b+c} \frac{\left(y^{|c|}-y^{-|c|}\right)\left(y^{|a|+b}-y^{-|a|-b}\right)}{(y-1 / y)^{2}}
$$

If instead $\zeta_{1}<0$, then $\phi_{21} \neq 0$ defines a point in $\mathbb{P}^{|a|-1}$, fibered over $\mathbb{P}^{|c|+b-1}$ parametrized by $\left(\phi_{13}, \phi_{23}\right) \neq(0,0)$, with index

$$
\chi_{Q}^{-}(y)=(-1)^{a+b+c} \frac{\left(y^{|a|}-y^{-|a|}\right)\left(y^{|c|+b}-y^{-|c|-b)}\right.}{(y-1 / y)^{2}}
$$

The difference

$$
\chi_{Q}^{+}(y)-\chi_{Q}^{-}(y)=(-1)^{a+c-1} \frac{y^{|c|-a}-y^{-|c|+a}}{y-1 / y} \times(-1)^{b-1} \frac{y^{b}-y^{-b}}{y-1 / y}
$$


is interpreted as the contribution of a two-particle bound state with charges $\left\{\gamma_{1+2}, \gamma_{3}\right\}$ [22].

Let us see how these results can be recovered from the localization formula (3.1). For vanishing $\mathrm{R}$-charges or flavor fugacities, the index is given by a residue of

$$
Z_{Q}=\left(\frac{y u_{1}-u_{2} / y}{u_{2}-u_{1}}\right)^{|a|}\left(\frac{y u_{3}-u_{2} / y}{u_{2}-u_{3}}\right)^{|b|}\left(\frac{y u_{3}-u_{1} / y}{u_{1}-u_{3}}\right)^{c} \widetilde{\prod_{i=1 \ldots 3}} \frac{\mathrm{d} u_{i}}{u_{i}(y-1 / y)}
$$

at the degenerate intersection $u_{1}=u_{2}=u_{3}$. The flag $\left(Q_{23}, Q_{12}\right)$, or equivalently $\left(Q_{23}, Q_{13}\right)$, has a degenerate matrix $\kappa_{F}$ so does not contribute for generic values of the $\zeta_{i}$ 's. For $\zeta_{1}<0, \zeta_{2}>0, \zeta_{3}<0$, the only stable flag is $\left(Q_{12}, Q_{23}\right) \sim\left(Q_{12}, Q_{13}\right)$, reproducing (4.10). For $\zeta_{1}>0, \zeta_{2}>0, \zeta_{3}<0$, the only stable flag is instead $\left(Q_{13}, Q_{12}\right) \sim\left(Q_{13}, Q_{23}\right)$, reproducing (4.9). For generic R-charges $R_{21}, R_{23}, R_{13}$, the degenerate intersection at $(0,0,0)$ splits into three non-degenerate intersections $p_{1}=H_{31} \cap H_{12}, p_{2}=H_{12} \cap H_{23}, p_{3}=H_{23} \cap H_{31}$. We denote by $p_{2}^{+}=\left(Q_{12}, Q_{23}\right)$ and $p_{2}^{-}=\left(Q_{23}, Q_{12}\right)$ the two possible flags at $p_{2}$, which are no longer equivalent, and similarly for $p_{3}^{ \pm}, p_{1}^{ \pm}$. Depending on $\zeta_{1}$, there are now two stable flags, given in the following table,

\begin{tabular}{|c|c|c|c|}
\hline$\zeta_{1}<\zeta_{3}<0$ & $\zeta_{3}<\zeta_{1}<0$ & $0<\zeta_{1}<\zeta_{2}$ & $0<\zeta_{2}<\zeta_{1}$ \\
\hline$\left\{p_{1}^{-}, p_{2}^{+}\right\}$ & $\left\{p_{1}^{-}, p_{2}^{-}\right\}$ & $\left\{p_{1}^{+}, p_{3}^{+}\right\}$ & $\left\{p_{1}^{+}, p_{3}^{-}\right\}$ \\
\hline
\end{tabular}

In each interval, the contribution of each flag is a complicated rational function of $y^{ \pm R_{i j}}$, but they combine in the same result (4.9) or (4.10), depending on the sign of $\zeta_{1}$. For generic flavor fugacities, the intersections become non-degenerate, and are in one-to-one correspondence with the possible fixed points. For those, only 2 out of the 3 set of arrows can be non-vanishing, leading to a total of either $|c|(|a|+b)$ or $|a|(b+|c|)$ fixed points, depending on the sign of $\zeta_{1}$. This agrees with (4.9) and (4.10) in the limit $y \rightarrow 1$.

\subsubsection{Four node quiver}

Next, we consider an example will become relevant in section 5.2: an Abelian quiver with 4 nodes $V_{1,2,3,4}$ and arrows $\kappa_{12}=a>0, \kappa_{23}=b<0, \kappa_{34}=c>0, \kappa_{41}=d<0$, which we denote by $Q=C_{a, b, c, d}$ :

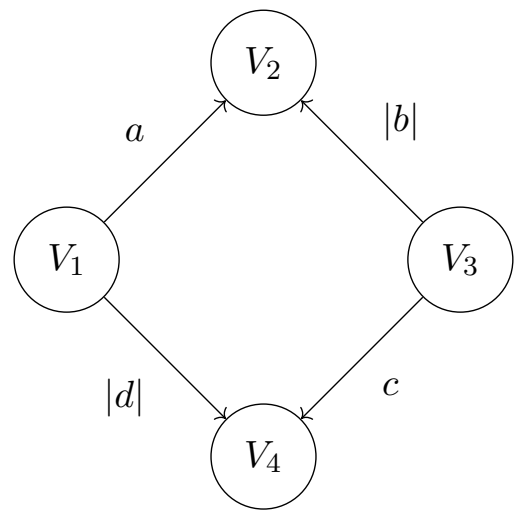

The D-term equations

$$
\begin{aligned}
\left|\phi_{12}\right|^{2}+\left|\phi_{14}\right|^{2}=\zeta_{1}, & -\left|\phi_{12}\right|^{2}-\left|\phi_{32}\right|^{2}=\zeta_{2}, \\
\left|\phi_{32}\right|^{2}+\left|\phi_{34}\right|^{2}=\zeta_{3}, & -\left|\phi_{14}\right|^{2}-\left|\phi_{34}\right|^{2}=\zeta_{4}
\end{aligned}
$$


admit solutions only when $\zeta_{1}, \zeta_{3}>0$ and $\zeta_{2}, \zeta_{4}<0$. Using the symmetries exchanging $\left(V_{1}, V_{3}\right)$ and $\left(V_{2}, V_{4}\right)$, there is no loss of generality in assuming that $c_{1}<c_{3}, c_{2}<c_{4}$. For vanishing $\mathrm{R}$-charges and flavor fugacities, the index is given by a residue of

$$
\begin{aligned}
Z_{Q}= & \left(\frac{y u_{2}-u_{1} / y}{u_{1}-u_{2}}\right)^{a}\left(\frac{y u_{2}-u_{3} / y}{u_{3}-u_{2}}\right)^{|b|}\left(\frac{y u_{4}-u_{3} / y}{u_{3}-u_{4}}\right)^{c}\left(\frac{y u_{4}-u_{1} / y}{u_{1}-u_{4}}\right)^{|d|} \\
& \times \prod_{i=1 \ldots . .4} \frac{\mathrm{d} u_{i}}{u_{i}(y-1 / y)}
\end{aligned}
$$

at the degenerate intersection $u_{1}=u_{2}=u_{3}=u_{4}$. For $\zeta_{2}+\zeta_{3}>0$, we find a single stable flag $\left(Q_{32}, Q_{34}, Q_{12}\right)$ contributing

$$
\chi_{Q}^{+}=(-1)^{a+b+c+d+1} \frac{\left(y^{|b|}-y^{-|b|}\right)\left(y^{c}-y^{-c}\right)\left(y^{a+|d|}-y^{-a-|d|}\right)}{(y-1 / y)^{3}}
$$

If instead $\zeta_{2}+\zeta_{3}<0$, there is a single stable flag $\left(Q_{32}, Q_{12}, Q_{34}\right)$ contributing

$$
\chi_{Q}^{-}=(-1)^{a+b+c+d+1} \frac{\left(y^{a}-y^{-a}\right)\left(y^{|b|}-y^{-|b|}\right)\left(y^{c+|d|}-y^{-c-|d|}\right)}{(y-1 / y)^{3}}
$$

The difference between the two contributions

$$
\chi_{Q}^{+}-\chi_{Q}^{-}=(-1)^{a+b+c+d} \frac{\left(y^{a-c}-y^{c-a}\right)\left(y^{|b|}-y^{-|b|}\right)\left(y^{|d|}-y^{-|d|}\right)}{(y-1 / y)^{3}}
$$

is recognized as the index of a bound state of $\gamma_{L}=\gamma_{1}+\gamma_{4}$ and $\gamma_{R}=\gamma_{2}+\gamma_{3}$. In the presence of generic R-charges, the degenerate intersection splits into 4 non-degenerate intersections, with 2 stable flags contributing in any chamber of the $\left(\zeta_{2}, \zeta_{3}\right)$ plane (for fixed values of $\zeta_{1}, \zeta_{4}$ satisfying the previous assumptions). For generic flavor fugacities (and vanishing $\mathrm{R}$-charges), the degenerate intersection splits into $a|b| c+a|b d|+a c|d|+c|b d|$ nondegenerate intersections, grouped in 4 subsets corresponding to the 4 possible spanning trees. Depending on the stability conditions, only two of these subsets support a stable flag contributing \pm 1 in the limit $y \rightarrow 1$, in agreement with the previous answers (4.16), (4.18).

\subsection{Abelian quivers with oriented loops}

In the presence of oriented loops and absence of a superpotential, the the quiver moduli space $\mathcal{M}_{Q}$ is in general non-compact. If one naively tries to apply the residue formula (3.1) for vanishing $\mathrm{R}$-charges and flavor fugacities, the intersections are typically degenerate and non-projective. One way to resolve this problem is to switch on generic flavor fugacities $\theta_{A}$ for the chiral fields; the resulting equivariant index is then a rational function of $y$ and $\nu_{A}=e^{\mathrm{i} \hbar \theta_{A}}$, with no natural way of taking the limit $\theta_{A} \rightarrow 0$. Another way out is to allow for a generic superpotential, by tuning the R-charges $R_{a b}$ such that the gauge invariant product of chiral fields around any oriented loops carries R-charge two. Additionally, one may switch on some flavor fugacities so as to restrict the form of the superpotential, but possibly at the cost of opening non-compact directions. 
Let us demonstrate this in the case of an Abelian quiver $Q=C_{a, b, c}$ with 3 nodes $V_{1}, V_{2}, V_{3}$ with an oriented loop, $a=\gamma_{12}>0, b=\gamma_{23}>0, c=\gamma_{31}>0$ :

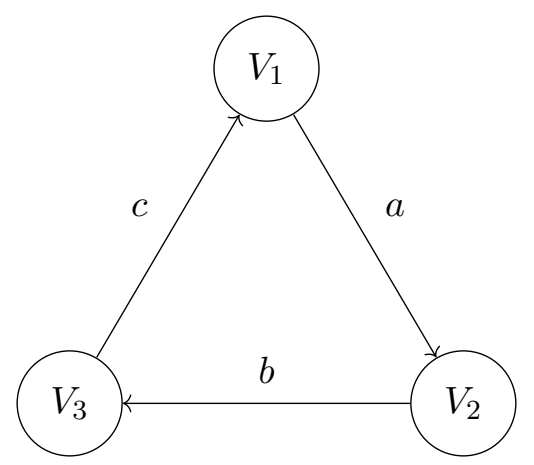

Choosing $R_{12}=R_{23}=R_{31}=2 / 3$ so that the total $\mathrm{R}$-charge of the loop is 2 , the index is given by a sum of residues of

$$
Z_{Q}=\left(\frac{y^{1 / 3} u_{2}-u_{1} / y}{u_{1}-y^{-2 / 3} u_{2}}\right)^{a}\left(\frac{y^{1 / 3} u_{3}-u_{2} / y}{u_{2}-y^{-2 / 3} u_{3}}\right)^{b}\left(\frac{y^{1 / 3} u_{1}-u_{3} / y}{u_{3}-y^{-2 / 3} u_{1}}\right)^{c} \widetilde{\prod_{i=1 \ldots 3}} \frac{\mathrm{d} u_{i}}{u_{i}(y-1 / y)}
$$

Denoting $H_{12}=u_{1}-y^{-2 / 3} u_{2}, H_{23}=u_{2}-y^{-2 / 3} u_{3}, H_{31}=u_{3}-y^{-2 / 3} u_{1}$ the singular hyperplanes, and $Q_{12}, Q_{23}, Q_{31}$ the corresponding charge vectors, we find three non-degenerate intersections $p_{2}=H_{12} \cap H_{23}, p_{3}=H_{23} \cap H_{31}, p_{1}=H_{31} \cap H_{12}$. We denote by $p_{2}^{+}$the flag $\left(Q_{12}, Q_{23}\right)$, and by $p_{2}^{-}$the flag $\left(Q_{23}, Q_{12}\right)$, and similarly for $p_{3}^{ \pm}, p_{1}^{ \pm}$. A single flag contributes for any signs of $\zeta_{1}, \zeta_{2}, \zeta_{3}$, given in the following table:

\begin{tabular}{|c|c|c|c|}
\hline$\zeta_{1}$ & $\zeta_{2}$ & $\zeta_{3}$ & $p$ \\
\hline- & - & + & $p_{1}^{+}$ \\
+ & - & + & $p_{1}^{-}$ \\
+ & - & - & $p_{2}^{+}$ \\
+ & + & - & $p_{2}^{-}$ \\
- & + & - & $p_{3}^{+}$ \\
- & + & + & $p_{3}^{-}$ \\
\hline
\end{tabular}

The corresponding residue of course gives the same result as the computation based on the identification of the quiver moduli space as a complete intersection in a product of projective spaces $[17,39]$, since both rely on the same index theorem.

Across the wall at $\zeta_{3}=0$ (say), assuming $\zeta_{1}>0, \zeta_{2}<0$, the flag $p_{1}^{-}$contributes when $\zeta_{3}>0$, while $p_{2}^{+}$contributes when $\zeta_{3}<0$. Defining $Z_{1}\left(u_{2}, u_{3}\right)=\operatorname{Res}_{u_{1}=y^{-2 / 3} u_{2}} Z_{Q}$, the difference of indices across the wall gives

$$
\Delta \chi=\operatorname{Res}_{u_{2}=y^{4 / 3} u_{3}} Z_{1}+\operatorname{Res}_{u_{2}=y^{-2 / 3} u_{3}} Z_{1}=-\left(\operatorname{Res}_{u_{2}=0}+\operatorname{Res}_{u_{2}=\infty}\right) Z_{1} .
$$

The integrand $Z_{1}\left(u_{2}, u_{3}\right)$ is of the form $f\left(u_{2}, u_{3}\right) \frac{\mathrm{d} u_{2}}{u_{2}}$, where $f\left(u_{2}, u_{3}\right)$ is a rational function of degree 0 . By homogeneity, the residues at $u_{2}=0$ and $u_{2}=\infty$ can be traded for residues 
at $u_{3}=\infty$ and $u_{3}=0$, respectively. The latter are easily computed from the limits

$$
\begin{gathered}
f\left(u_{2}, u_{3}\right) \stackrel{u_{3} \rightarrow 0}{\rightarrow} \frac{(-y)^{b-c}}{(y-1 / y)^{2}} \oint_{y^{-2 / 3} u_{2}} \frac{\mathrm{d} u_{1}}{u_{1}}\left(\frac{y^{1 / 3} u_{2}-u_{1} / y}{u_{1}-y^{-2 / 3} u_{2}}\right)^{a}=(-1)^{a+b+c} \frac{y^{b-c}\left(y^{a}-y^{-a}\right)}{(y-1 / y)^{2}} \\
f\left(u_{2}, u_{3}\right) \stackrel{u_{3} \rightarrow \infty}{\rightarrow} \frac{(-y)^{c-b}}{(y-1 / y)^{2}} \oint_{y^{-2 / 3} u_{2}} \frac{\mathrm{d} u_{1}}{u_{1}}\left(\frac{y^{1 / 3} u_{2}-u_{1} / y}{u_{1}-y^{-2 / 3} u_{2}}\right)^{a}=(-1)^{a+b+c} \frac{y^{c-b}\left(y^{a}-y^{-a}\right)}{(y-1 / y)^{2}}
\end{gathered}
$$

leading to

$$
\Delta \chi=(-1)^{b+c-1} \frac{y^{c-b}-y^{b-c}}{y-1 / y} \times(-1)^{a-1} \frac{y^{a}-y^{-a}}{y-1 / y}
$$

which is recognized as the contribution from the bound state with charges $\left\{\gamma_{1}+\gamma_{2}, \gamma_{3}\right\}$. This computation illustrates how discontinuities across the wall come from poles at infinity [31].

Let us now consider the attractor point (3.9) where $\zeta_{1}^{*}=c-a, \zeta_{2}^{*}=a-b, \zeta_{3}^{*}=b-c$. For $a, b<c$, the contribution comes from the flag $p_{2}^{+}$if $b>a$, or from the flag $p_{2}^{-}$if $a<b$. The residue associated to $p_{i}^{+}$and $p_{i}^{-}$coincide, since there are only two hyperplanes intersecting at $p_{i}$. The result should be compared with the Coulomb branch formula evaluated at the attractor point,

$$
\Omega_{*}(1,1,1)=\Omega_{\mathrm{S}}(1,1,1)+g_{C}\left(\left\{\gamma_{1}, \gamma_{2}, \gamma_{3}\right\}\right)+H\left(\left\{\gamma_{1}, \gamma_{2}, \gamma_{3}\right\},\{1,1,1\}\right)
$$

where $\Omega_{\mathrm{S}}(1,1,1)$ is the single-centered invariant, and the modified Coulomb index is given by $[17, \S 3.3]$

$$
\begin{aligned}
g_{C}\left(\left\{\gamma_{1}, \gamma_{2}, \gamma_{3}\right\}\right) & =(-1)^{k} \frac{y^{k}+y^{-k}}{(y-1 / y)^{2}} \\
H\left(\left\{\gamma_{1}, \gamma_{2}, \gamma_{3}\right\},\{1,1,1\}\right) & = \begin{cases}-2(y-1 / y)^{-2} & k \text { even } \\
(y+1 / y)(y-1 / y)^{-2} & k \text { odd }\end{cases}
\end{aligned}
$$

with $k=c-a-b$ (more generally, $k$ is equal to the largest of $a, b, c$ minus the sum of the other two). Evaluating the l.h.s. of (4.24) via the residue formula allows to read off the single-centered invariant $\Omega_{\mathrm{S}}(1,1,1)$. Note that both $\Omega_{\mathrm{S}}(1,1,1)$ and $g_{C}+H$ vanish unless $c \leq a+b-2$, although $g_{C}$ by itself does not vanish for $a+b-2<c \leq a+b-c$. This is consistent with the fact that the quiver moduli space, when non-empty, has complex dimension $d=a+b-c-2$.

It is instructive to compare the index in the presence of a generic superpotential, computed using an assignment of R-charges such that the oriented loop carries charge 2 , to the index for vanishing superpotential. As explained in $[17, \S 2.5]$, the latter can be computed using the Harder-Narasimhan recursion, or equivalently Reineke's formula. Either way, one finds, in the chamber $\zeta_{1}>\zeta_{2}>0, \zeta_{3}<0$,

$$
\chi_{Q}^{W=0}=(-1)^{a+b+c} y^{2-a-b-c}\left(1-y^{2 a}\right)\left(1-y^{2 b}\right) /\left(1-y^{2}\right)^{2}
$$

which in particular is not invariant under $y \rightarrow 1 / y$. This is consistent with the fact that the quiver moduli space with vanishing superpotential is a $\mathbb{P}^{a-1} \times \mathbb{P}^{b-1}$ bundle over 
the non-compact base $\mathbb{C}^{c}[17, \S 3.3]$. The effect of the superpotential is to restrict to the point at the origin in $\mathbb{C}^{c}$, and to a complete intersection of $c$ hypersurfaces in the fiber over that point. The first operation removes a factor $y^{-c}$ in the index, and the second, by virtue of the Lefschetz hyperplane theorem, multiplies by an additional factor of $y^{c}$, as far as only negative powers of $y$ are concerned. The result of these two operations, $(-1)^{a+b+c} y^{2+c-a-b}\left(1-y^{2 a}\right)\left(1-y^{2 b}\right) /\left(1-y^{2}\right)^{2}$ agrees with the Coulomb index (4.25) up to positive powers of $y$, which therefore correctly captures the non-middle part of the cohomology, while the middle part is captured by the single-centered invariant $\Omega_{\mathrm{S}}(1,1,1)$.

Here we note that the same result (4.27) can be obtained by applying the formula (3.1) for vanishing $\mathrm{R}$-charge but generic flavor potentials $\nu_{1 A}(A=1, \ldots a), \nu_{2 B}(B=1, \ldots b)$, $\nu_{3 C}(C=1, \ldots c)$, which requires extracting a suitable residue of

$$
\begin{aligned}
Z_{Q}= & \prod_{A=1}^{a}\left(\frac{u_{2} y \nu_{1 A}-u_{1} / y}{u_{1}-u_{2} \nu_{1 A}}\right) \prod_{B=1}^{b}\left(\frac{u_{3} y \nu_{2 B}-u_{2} / y}{u_{2}-u_{3} \nu_{2 B}}\right) \prod_{C=1}^{c}\left(\frac{u_{1} y \nu_{3 C}-u_{3} / y}{u_{3}-u_{1} \nu_{3 C}}\right) \\
& \times \widetilde{\prod_{i=1 \ldots 3}} \frac{\mathrm{d} u_{i}}{u_{i}(y-1 / y)}
\end{aligned}
$$

Denoting $H_{12, A}=u_{1}-u_{2} \nu_{1 A}$, etc the singular hyperplanes, there are $a \times b$ non-degenerate intersections $H_{12, A_{0}} \cap H_{23, B_{0}}, b \times c$ intersections $H_{2 B_{0}} \cap H_{31, C_{0}}$ and $a \times c$ intersections $H_{12, A_{0}} \cap H_{31, C_{0}}$. In the chamber $c_{1}>0, c_{2}<0, c_{3}<0$, only the flags $\left(Q_{23, B_{0}}, Q_{12, A_{0}}\right)$ contribute, each of them giving

$$
\begin{aligned}
\prod_{A \neq A_{0}}^{a}\left(\frac{y \nu_{1 A}-\nu_{1 A_{0}} / y}{\nu_{1 A_{0}}-\nu_{1 i}}\right) \prod_{B \neq B_{0}}^{b}( & \left.\frac{y \nu_{2 B}-\nu_{2 B_{0}} / y}{\nu_{2 B_{0}}-\nu_{2 j}}\right) \\
& \times \prod_{C=1}^{c}\left(\frac{y \nu_{1 A_{0}} \nu_{2 B_{0}} \nu_{3 C}-1 / y}{1-\nu_{1} A_{0} \nu_{2} B_{0} \nu_{3 C}}\right) \stackrel{y \longrightarrow 1}{\longrightarrow}(-1)^{a+b+c}
\end{aligned}
$$

Summing over all choices of $A_{0} B_{0}$ and taking the limit $y \rightarrow 1$, we obtain $(-1)^{a+b+c} a b$ in agreement with (4.27). Keeping $y \neq 1$, we recognize in (4.29) the contribution of the fixed point under the flavor rotation $\varphi_{i, A} \mapsto e^{\mathrm{i} \hbar \theta_{i, A}}$ given by $P: \phi_{12, A}=\delta_{A, A_{0}}, \phi_{23, B}=$ $\delta_{B, B_{0}}, \phi_{31, C}=0$. The flavor rotation act on the tangent space by a compensating gauge rotation,

$$
\Phi_{1 A} \mapsto e^{\mathrm{i}\left(\theta_{1 A}+\phi_{1}-\phi_{2}\right)} \Phi_{1 A}, \quad \Phi_{2 B} \mapsto e^{\mathrm{i}\left(\theta_{2 B}+\phi_{2}-\phi_{3}\right)} \Phi_{2 B}, \quad \Phi_{3 C} \mapsto e^{\mathrm{i}\left(\theta_{3 C}+\phi_{3}-\phi_{1}\right)} \Phi_{3 C},
$$

with $\theta_{1 A_{0}}+\phi_{1}-\phi_{2}=0$ and $\theta_{2 B_{0}}+\phi_{2}-\phi_{3}=0$, so that its determinant reproduces the denominator in (4.29), in agreement with (3.8),

$$
\operatorname{det}_{\mathbb{C}}\left(1-\mathrm{d} f_{P}\right)=\prod_{A \neq A_{0}}\left(1-\nu_{1 A} / \nu_{1 A_{0}}\right) \prod_{B \neq B_{0}}\left(1-\nu_{2 B} / \nu_{2} B_{0}\right) \prod_{C}\left(1-\nu_{3 C} \nu_{1 A_{0}} \nu_{2 B_{0}}\right) .
$$

Rescaling the fugacities as $\nu_{1, A} \rightarrow t^{A} \nu_{1, A}, \nu_{2, B} \rightarrow t^{B} \nu_{1, B}, \nu_{3, C} \rightarrow t^{C} \nu_{3, C}$ and then take the limit $t \rightarrow \infty$, then each of the $a b$ contributions (4.29) produces a single power of $y$, which sum up to the same result (4.27) as obtained from Reineke's formula. 


\section{Non-Abelian quivers}

In this section, we finally turn to non-Abelian quivers, and demonstrate how the residue formula (3.1) combined with the Cauchy-Bose identity (3.12) gives a natural decomposition of the index in terms according to partitions of the total dimension vector, in agreement with the Coulomb branch formula.

\subsection{Kronecker quiver with $\operatorname{rank}(N, 1)$}

We consider a quiver $Q=K_{m}$ with two nodes $V_{1}, V_{2}$ and $m>0$ arrows from $V_{1}$ to $V_{2}$ :

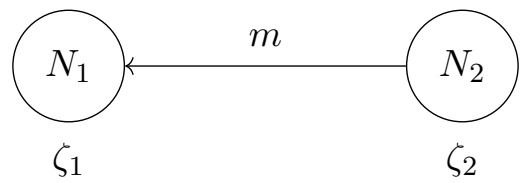

We choose the dimension vector $\left(N_{1}, N_{2}\right)=(N, 1)$ and stability parameters $\zeta_{1}<0, \zeta_{2}>0$ such that $N \zeta_{1}+\zeta_{2}=0$. For $m \geq N$, the quiver moduli space $\mathcal{M}_{Q}$ is known to be the Grassmannian $G(N, m)$ of $N$-dimensional planes inside $\mathbb{C}^{m}$, of dimension $d=N(m-N)$, with $\chi$-genus given by

$$
\chi_{Q}(y)=\frac{(-y)^{-N(m-N)}[m, y] !}{[N, y] ![m-N, y] !}
$$

where $[m, y] !=\prod_{k=1}^{m}\left(1-y^{2 k} /\left(1-y^{2}\right)\right.$ is the deformed factorial. For $m<N$, the moduli space is empty. Our aim is to rederive this well-known result using localization, and explain how the decomposition predicted by the Coulomb branch formula naturally emerges in this context.

In order to apply the localization formula (3.1), we upgrade the stability vector $\zeta=$ $\left(\zeta_{1}, \zeta_{2}\right)$ to $\eta=\left(\eta_{1}, \ldots, \eta_{N}, \zeta_{2}\right)$ where $\eta_{1}<\eta_{2}<\cdots<\eta_{N}$ and $\eta_{s} \simeq \zeta_{1}$ for $s=1 \ldots N$. We denote by $\left(v_{1}, \ldots, v_{N}\right)$ and $u$ the exponentiated Cartan variables associated to the vertices $V_{1}$ and $V_{2}$. The equivariant $\chi$-genus is then a sum of residues of

$$
Z_{Q}=\left(\prod_{s \neq s^{\prime}} \frac{u_{s^{\prime}}-u_{s}}{u_{s} / y-y u_{s^{\prime}}}\right) \prod_{s=1}^{N}\left(\prod_{A=1}^{m} \frac{y \nu_{A} u_{s}-u / y}{u-\nu_{A} u_{s}} \frac{\mathrm{d} u_{s}}{u_{s}(y-1 / y)}\right)
$$

As argued in $[51,52]$, residues involving vector multiplet poles $u_{s} / y-y u_{s^{\prime}}$ always vanish, so the only contribution comes from the intersection of the hyperplanes $\left(u-\nu_{A_{1}} u_{1}, u-\right.$ $\left.\nu_{A_{2}} u_{2}, \ldots u-\nu_{A_{N}} u_{N}\right)$, for any subset of $N$ distinct elements $\left\{A_{1}, \ldots, A_{N}\right\} \subset\{1, \ldots, m\}$, taken in this particular order for the above choice of $\eta$. In this way one arrives at ${ }^{9}$

$$
\chi_{Q}\left(y, \nu_{A}\right)=\frac{1}{N !} \oint Z_{Q}=\sum_{\mathcal{I} \in C(N, m)} \prod_{A \in \mathcal{I}} \prod_{B \notin \mathcal{I}} \frac{\nu_{A} / y-\nu_{B} y}{\nu_{A}-\nu_{B}}
$$

where $\mathcal{I}$ runs over all subsets of $N$ distinct elements $\left\{A_{1}, \ldots, A_{N}\right\} \subset\{1, \ldots, m\}$. Each term in this sum originates from a $\mathrm{U}(1)$ fixed point on $G(N, m)$, where the $N \times m$ matrix $\phi_{s A}$

\footnotetext{
${ }^{9}$ This agrees with $[28, \S 4.6]$, upon taking the limit $\tau \rightarrow \mathrm{i} \infty$ in their formula.
} 
has $N$ non-zero elements in positions $\left(s, A_{s}\right)$. The result can be shown to be independent ${ }^{10}$ of the fugacities $\nu_{A}$, and equal to the deformed binomial coefficient (5.1).

In order to explain the connection with the Coulomb branch formula, we set to one the flavor fugacities $\nu_{A}$ and fix the gauge $u=1$, obtaining

$$
\chi_{Q}(y)=\frac{1}{(y-1 / y)^{N} N !} \int \prod_{s=1}^{N} \frac{\mathrm{d} u_{s}}{2 \pi \mathrm{i} u_{s}} \prod_{s \neq s^{\prime}} \frac{u_{s^{\prime}}-u_{s}}{u_{s} / y-y u_{s^{\prime}}} \prod_{s=1}^{N}\left(\frac{y u_{s}-1 / y}{1-u_{s}}\right)^{m}
$$

where the integral runs over a product of small circles around $u_{s}=1$. Using the CauchyBose identity (3.12), we can rewrite (5.4) as a sum over permutations,

$$
\chi_{Q}=\frac{(-1)^{N}}{N !} \int \prod_{s=1}^{N} \frac{\mathrm{d} u_{s}}{2 \pi \mathrm{i}} \sum_{\sigma \in S_{N}} \frac{\epsilon(\sigma)}{\prod_{s=1}^{N}\left(u_{s} / y-y u_{\sigma(s)}\right)} \prod_{s=1}^{N}\left(\frac{y u_{s}-1 / y}{1-u_{s}}\right)^{m}
$$

Decomposing each permutation $\sigma$ into a product of cycles $\prod_{\ell}\left(\mathcal{C}_{\ell}\right)^{n_{\ell}}$, the integral factorizes into a product of factors associated to each cycle $\mathcal{C}_{\ell}$ of length $\ell$,

$$
\chi_{Q}=\frac{(-1)^{N}}{N !} \sum_{\sigma \in S_{N}} \epsilon(\sigma) \prod_{\ell}\left(\mathcal{I}_{\ell}\right)^{n_{\ell}}
$$

where $\mathcal{I}_{\ell}$ denotes the integral (with $x_{i} \equiv x_{i+L}$ ),

$$
\mathcal{I}_{\ell}=\prod_{i=1}^{\ell} \oint_{1} \frac{\mathrm{d} x_{i}}{2 \pi \mathrm{i}} \frac{1}{\prod_{i=1}^{\ell}\left(y x_{i}-x_{i+1} / y\right)}\left(\frac{y x_{i}-1 / y}{x_{i}-1}\right)^{m}
$$

Moreover, the signature of the permutation is $\epsilon(\sigma)=\prod_{\ell}(-1)^{n_{\ell}(\ell-1)}$. By successively integrating over each $u_{i}$, one can establish that

$$
\mathcal{I}_{\ell}=\frac{y^{m \ell}-y^{-m \ell}}{y^{\ell}-y^{-\ell}}
$$

For this, one may deform the contour around $x_{i}=1$ to a sum of contours around $x_{i}=$ $x_{i+1} / y^{2}$ and $x_{i}=y^{2} x_{i-1}$. The residue at $x_{i}=x_{i+1} / y^{2}$ is regular at $x_{i+1}=1$ (due to a cancellation of factors of $\left(x_{i+1}-1\right)^{m}$ in the nominator and denominator), so can be dropped. The residue at $x_{i}=y^{2} x_{i-1}$ produces a factor $\left[\left(y^{2} x_{i-1}-1 / y^{2}\right) /\left(y x_{i-1}-1 / y\right)\right]^{m}$, which cancels partially against $\left[\left(y x_{i-1}-1 / y\right) /\left(x_{i-1}-1\right)\right]^{m}$, etc. Alternatively, one may reinstate the flavor fugacities and evaluate

$$
\mathcal{I}_{\ell}=\int \prod_{i=1}^{\ell} \frac{\mathrm{d} x_{i}}{2 \pi \mathrm{i}} \frac{1}{\prod_{i=1}^{\ell}\left(x_{i} / y-y x_{i+1}\right)} \prod_{A=1}^{m} \frac{y \nu_{A} x_{i}-1 / y}{1-\nu_{A} x_{i}}
$$

where the integral circles around each pole at $x_{i}=\nu_{A(i)}$ for all maps $A:[1, \ell] \rightarrow[1, m]$. In the limit $y \rightarrow 1$ it is easy to check that constant maps contribute +1 while non-constant maps contribute 0 , leading to $\mathrm{i}_{\ell} \rightarrow m$. For $y \neq 1$ but assuming $\nu_{1} \ll \cdots \ll \nu_{m}$, one

\footnotetext{
${ }^{10}$ For this it suffices to show that the residue at the potential singularities $\nu_{A}=\nu_{B}$ vanish.
} 
also finds that the only non-vanishing contribution come from constant maps $A(i)=A$, contributing $y^{\ell(2 A-m-1)}$, in agreement with (5.8).

Using (5.8), and noting that the number of permutations with cycle shape $\lambda=\sum \ell n_{\ell}$ is $N ! / \prod_{\ell} \ell^{n_{\ell}} n_{\ell} !$, one may rewrite (5.6) as a sum over partitions of $N$,

$$
\chi_{Q}=(-1)^{N(m+1)} \sum_{N=\sum_{\ell=1}^{N}} \prod_{\ell n_{\ell}}^{N} \frac{\left[(-1)^{\ell+1} \mathcal{I}_{\ell}\right]^{n_{\ell}}}{\ell^{n_{\ell}} n_{\ell} !}=\sum_{N=\sum_{\ell=1}^{N}} \prod_{\ell n_{\ell}}^{N} \frac{\left[(-1)^{m \ell+1} \mathcal{I}_{\ell}\right]^{n_{\ell}}}{\ell^{n_{\ell}} n_{\ell} !}
$$

Note that this coincides with the cycle index $Z_{N}\left(\left\{t_{\ell}\right\}\right)$ for the permutation group $S_{N}$, evaluated at $t_{\ell}=(-1)^{m \ell+1} \mathcal{I}_{\ell}$. In order to evaluate the sum over partitions, it is expedient to construct the generating function,

$$
G=\sum_{N=0}^{\infty} Z_{N}\left(\left\{t_{\ell}\right\}\right) t_{0}^{N}=\exp \left(-\sum_{\ell=1}^{\infty} \frac{(-y)^{m \ell}-(-y)^{-m \ell}}{\ell\left(y^{\ell}-y^{-\ell}\right)} t_{0}^{\ell}\right) .
$$

The result agrees with $Z_{\text {halo }}$ in [24, (4.72)], up to a change $m \rightarrow-m$ since we have $m>0$ while the result of loc.cit. assumed $m<0$.

We can now compare (5.10) with the Coulomb branch formula (2.5) for this system (or equivalently (2.9)),

$$
\chi_{Q}=\sum_{N=\sum_{\ell=1}^{N} \ell n_{\ell}} \frac{g_{C}\left(\left\{n_{1} \times \gamma_{1}, n_{2} \times 2 \gamma_{1}, \ldots n_{\ell} \times \ell \gamma_{1}, \gamma_{2}\right\}\right)}{\prod_{\ell=1}^{N} n_{\ell} !} \prod_{\ell=1}^{N}\left[\bar{\Omega}_{S}\left(\ell \gamma_{1}\right)\right]^{n_{\ell}} \bar{\Omega}_{S}\left(\gamma_{2}\right)
$$

where $n \times \ell \gamma$ denotes $n$ copies of the vector $\ell \gamma$. Since the only non-vanishing DSZ products are $\left\langle\ell \gamma_{1}, \gamma_{2}\right\rangle=m \ell$, the Coulomb index factorizes into

$$
g_{C}\left(n_{1} \times \gamma_{1}, n_{2} \times 2 \gamma_{1}, \ldots n_{\ell} \times \ell \gamma_{1}, \gamma_{2}\right)=\prod_{\ell=1}^{N}\left(\frac{y^{m \ell}-y^{-m \ell}}{y-1 / y}\right)^{n_{\ell}}
$$

which is also the $\chi$-genus of the Abelian quiver $Q\left(\left\{\gamma_{i}\right\}\right)$ described below (2.9). Moreover, in the absence of loops, the only non-vanishing single centered invariants are $\Omega_{S}\left(\gamma_{1}\right)=$ $\Omega_{S}\left(\gamma_{2}\right)=1$, hence

$$
\bar{\Omega}_{S}\left(\ell \gamma_{1}\right)=\frac{y-1 / y}{\ell\left(y^{\ell}-y^{-\ell}\right)}, \quad \bar{\Omega}_{S}\left(\ell \gamma_{2}\right)=1
$$

Combining these relations, we conclude that (5.10) is in perfect agreement with the Coulomb branch formula. Moreover, the sum over partitions of $\gamma=N \gamma_{1}+\gamma_{2}$ clearly originates from the sum over conjugacy classes in the permutation group $S_{N}$.

\section{$5.2 \quad$ Star quivers}

We now turn to a generalization of both the Abelian star quiver $S_{\left\{a_{i}\right\}}$ considered in section 4.1, and the Kronecker quiver $K_{m}$ of $\operatorname{rank}(N, 1)$ in the previous subsection. Namely, we consider a quiver with $K+1$ vertices $V_{0}, V_{1}, \ldots V_{K}$, with $a_{i}>0$ arrows from $V_{i}$ to $V_{0}$, and with dimension vector $(N, 1,1, \ldots)$. Our aim is to evaluate the index using the Cauchy-Bose formula for the contribution of the $\mathrm{U}(N)$ vector multiplets, and show that 
the resulting decomposition agrees with the Coulomb branch formula (2.5), or equivalently the MPS formula (2.9).

Clearly, the moduli space is trivial unless $\zeta_{0}<0$ and $\zeta_{i}>0$ for $i=1 \ldots K$. Up to relabelling the nodes, we can assume that $\zeta_{1}>\zeta_{2}>\cdots>\zeta_{K}$. We shall further assume that $\zeta_{1} \gg \zeta_{2} \gg \cdots \gg \zeta_{K}$, and upgrade the stability vector to $\eta=\left(\eta_{1}, \ldots, \eta_{N}, \zeta_{1}, \ldots \zeta_{K}\right)$ with $\eta_{1}<\eta_{2}<\cdots<\eta_{N}$ and $\eta_{s} \simeq \zeta_{0}$ for $s=1 \ldots N$. We denote by $\left(v_{1}, \ldots, v_{N}\right)$ and $\left(u_{1}, \ldots u_{K}\right)$ the exponentiated Cartan variables associated to the vertices $V_{0}$ and $V_{i}$. The $\chi$-genus is then a sum of residues of

$$
Z_{Q}=\frac{1}{(y-1 / y)^{N+K-1}}\left(\prod_{s \neq s^{\prime}} \frac{v_{s^{\prime}}-v_{s}}{v_{s} / y-y v_{s^{\prime}}}\right)\left(\prod_{\substack{s=1 \ldots N \\ i=1 \ldots K}}\left[\frac{y v_{s}-u_{i} / y}{u_{i}-v_{s}}\right]^{a_{i}}\right) \prod_{i=1}^{K} \frac{\mathrm{d} u_{i}}{u_{i}} \prod_{s=1 \ldots N} \frac{\mathrm{d} v_{s}}{v_{s}}
$$

As for the Grassmannian in the previous subsection, residues involving vector multiplet poles $v_{s} / y-y v_{s^{\prime}}$ always vanish [51,52], so the only contribution comes from the intersection of the $N K$ hyperplanes $u_{i}-v_{s}$, which is degenerate if $K \geq 2$. For the above choice of $\eta$, this intersection carries a single stable flag $\left(u_{1}-v_{1}, u_{1}-v_{2}, \ldots, u_{1}-v_{N}, u_{2}-v_{N}, u_{K}-v_{N}\right)$, corresponding to the following integration prescription

$$
\chi_{Q}=\frac{1}{N !} \oint Z_{Q}, \quad \oint:=(-1)^{N-1} \oint_{v_{N}} \frac{\mathrm{d} u_{K}}{u_{K}} \cdots \oint_{v_{N}} \frac{\mathrm{d} u_{1}}{u_{1}} \oint_{u_{1}} \frac{\mathrm{d} v_{N-1}}{v_{N-1}} \cdots \oint_{u_{1}} \frac{\mathrm{d} v_{1}}{v_{1}}
$$

We now apply the Cauchy-Bose formula (3.12) to the vector multiplet product in (5.15), and collect contributions according to the cycle shape of the permutation $\sigma \in S_{N}$, corresponding to a partition $\lambda=\ell_{1}+\cdots+\ell_{p}=\sum \ell n_{\ell}$, where $n_{\ell}$ is the number of $n_{i}$ 's equal to $\ell$ For convenience we relabel the Cartan variables for the non-Abelian group accordingly,

$$
\left\{v_{1}, \ldots, v_{N}\right\} \rightarrow\left\{v_{1}^{(1)}, \ldots, v_{\ell_{1}}^{(1)} ; v_{1}^{(2)}, \ldots, v_{\ell_{2}}^{(2)} ; \ldots ; v_{1}^{(p)}, \ldots, v_{\ell_{p}}^{(p)}\right\}
$$

All permutations with the same cycle shape $\lambda$ give the same contribution to the $\chi$-genus,

$$
\begin{aligned}
\chi_{\lambda}= & (-1)^{N-p}\left(y-y^{-1}\right)^{-N-K+1}(-1)^{N-1}\left(1-y^{2}\right)^{N} \\
& \times \oint_{v_{N}} \frac{\mathrm{d} u_{K}}{u_{K}} \cdots \oint_{v_{N}} \frac{\mathrm{d} u_{1}}{u_{1}} \oint_{u_{1}} \frac{\mathrm{d} v_{N-1}}{v_{N-1}} \cdots \oint_{u_{1}} \frac{\mathrm{d} v_{1}}{v_{1}} \\
& \times\left[\prod_{\beta=1}^{p} \frac{v_{1}^{(\beta)} \ldots v_{\ell_{\beta}}^{(\beta)}}{\left(v_{1}^{(\beta)}-y^{2} v_{2}^{(\beta)}\right) \ldots\left(v_{\ell_{\beta}}^{(\beta)}-y^{2} v_{1}^{(\beta)}\right)}\right] \prod_{s=1}^{N} \prod_{\alpha=1}^{K}\left(\frac{y v_{s}-y^{-1} u_{\alpha}}{u_{\alpha}-v_{s}}\right)^{a_{\alpha}} .
\end{aligned}
$$

where $v_{N}$ can be gauged fixed to any value. The sum over all permutations with the same cycle shape turn the prefactor $1 / N$ ! into a factor $1 /\left(\prod_{\ell} n_{\ell} ! !^{n_{\ell}}\right)$. According to the MPS formula (2.9), (5.18) should coincide with

$$
\tilde{\chi}_{\lambda}=\frac{\left(y-y^{-1}\right)^{p}}{\prod_{\beta=1}^{p}\left(y^{\ell_{\beta}}-y^{-\ell_{\beta}}\right)} \chi\left(Q_{\lambda}\right)
$$


where $Q_{\lambda}$ is the Abelian quiver

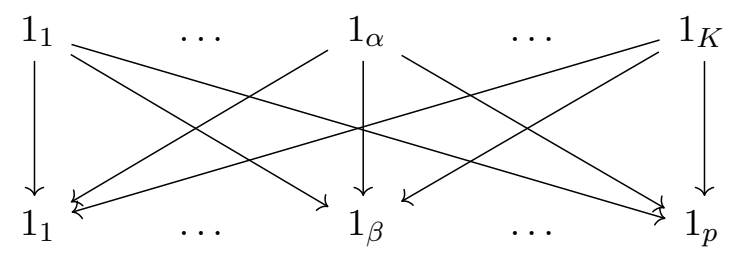

with $\ell_{\beta} \times a_{i}$ arrows going from the node $1_{i}$ on the top row to the node $1_{\beta}$ on the bottom row. The $\chi$-index for $Q_{\lambda}$ follows from the residue formula (3.1),

$$
\begin{aligned}
\tilde{\chi}_{\lambda}= & \frac{(-1)^{p-1}\left(y-y^{-1}\right)^{-K+1}}{\prod_{\beta=1}^{p}\left(y^{\ell_{\beta}}-y^{-\ell_{\beta}}\right)} \\
& \times \oint_{v_{p}} \frac{\mathrm{d} u_{K}}{u_{K}} \cdots \oint_{v_{p}} \frac{\mathrm{d} u_{1}}{u_{1}} \oint_{u_{1}} \frac{\mathrm{d} v_{p-1}}{v_{p-1}} \cdots \oint_{u_{1}} \frac{\mathrm{d} v_{1}}{v_{1}} \prod_{r=1}^{p}\left[\prod_{\alpha=1}^{K}\left(\frac{y v_{r}-y^{-1} u_{\alpha}}{u_{\alpha}-v_{r}}\right)^{a_{\alpha}}\right]^{\ell_{r}} .
\end{aligned}
$$

where $v_{p}$ can be gauge fixed to any value. We shall now prove, by induction on $K$, that (5.18) and (5.20) coincide. For $K=2$, this is the Kronecker quiver with rank $(N, 1)$ discussed in the previous subsection. We shall assume that the equality $\chi_{\lambda}=\tilde{\chi}_{\lambda}$ holds for star quivers with $K-1$ nodes, and show that it continues to hold for $K$ nodes.

To show that (5.18) and (5.20) coincide, we focus on the last contour integral over $u_{K}$ around $v_{N}$ in , (5.18), or around $v_{p}$ in (5.20), which we free to identify by a choice of gauge. Denoting by $\chi_{\Lambda}\left(u_{K}, v\right)$ and $\tilde{\chi}_{\Lambda}\left(u_{K}, v\right)$ the two integrands, we need to prove

$$
\oint_{v} \frac{\mathrm{d} u_{K}}{u_{K}}\left[\chi_{\Lambda}\left(u_{K}, v\right)-\tilde{\chi}_{\Lambda}\left(u_{K}, v\right)\right]=0,
$$

By construction, $\chi_{\Lambda}\left(u_{K}, v\right)$ and $\tilde{\chi}_{\Lambda}\left(u_{K}, v\right)$ are homogenous rational functions of degree 0 . The recursion shows that their difference can be put to the form

$$
\chi_{\Lambda}\left(u_{K}, v\right)-\tilde{\chi}_{\Lambda}\left(u_{K}, v\right)=\frac{N_{m}\left(u_{K}, v\right)}{\left(u_{K}-v\right)^{m}},
$$

where $m$ is a non-negative integer and $N_{m}\left(u_{K}, v\right)$ is a homogeneous polynomial of degree $m$. In particular, the denominator does not have any factor of $u_{K}$ or $v$. The equality (5.21) will follow if we can show that the numerator factorizes as

$$
N_{m}\left(u_{K}, v\right)=v^{m_{1}} u_{K}^{m_{2}} \tilde{N}_{m-m_{1}-m_{2}}
$$

with $m_{1}, m_{2} \geq 1$. Indeed, setting $v=1$ by homogeneity, it follows from (5.23) that

$$
\oint_{1} \frac{\mathrm{d} u_{K}}{u_{K}}\left[\chi_{\Lambda}\left(u_{K}, 1\right)-\tilde{\chi}_{\Lambda}\left(u_{K}, 1\right)\right]=\oint_{1} \mathrm{~d} u_{K} \frac{u_{K}^{m_{2}-1} \tilde{N}_{m-m_{1}-m_{2}}\left(u_{K}, 1\right)}{\left(u_{K}-1\right)^{m}}=0
$$

since the numerator has monomials of degree less than $m-1$ are annihilated by the $(m-1)$ differentiations needed to extract the residue. To show that $N_{m}\left(u_{K}, v\right)$ factorizes as in (5.23), it suffices to check that $I_{K}\left(u_{K}, v\right)$ vanishes both when $u_{K} \rightarrow 0$ and when $u_{K} \rightarrow \infty$ (which is equivalent to $v \rightarrow 0$ by homogeneity). In either of those limits, the integrands $\chi_{\lambda}\left(u_{K}, v\right)$ and $\tilde{\chi}_{\lambda}\left(u_{K}, v\right)$ reduce to the indices for the star quiver obtained by removing the node $v_{K}$. By induction, the equality $\chi_{\lambda}=\tilde{\chi}_{\lambda}$ therefore holds for star quivers with arbitrary number of Abelian nodes. 


\subsection{Kronecker quiver with rank $\left(N_{1}, N_{2}\right)$}

We now consider the Kronecker quiver $K_{m}$ introduced in section 5.1, now for general dimension vector. The $\chi$-genus of the moduli space $\mathcal{M}_{Q}$ has been computed in the mathematics literature $[42,53,54]$ using the Atiyah-Bott Lefschetz fixed point theorem, and in the physics literature $[32,51,52,55]$ using supersymmetric localization. Our goal here is to clarify the relation between the two approaches, and explain how the Coulomb branch formula arises from applying the Cauchy-Bose formula to both nodes.

The quiver moduli space $\mathcal{M}_{Q}$ is non-trivial in the chamber $\zeta_{1}<0<\zeta_{2}$ with $N_{1} \zeta_{0}+$ $N_{2} \zeta_{1}=0$, in which case its complex dimension is $d=m N_{1} N_{2}-N_{1}^{2}-N_{2}^{2}+1$. In order to apply the residue formula (3.1), we upgrade the stability vector $\zeta=\left(\zeta_{0}, \zeta_{1}\right)$ to $\eta=$ $\left(\eta_{1}, \ldots, \eta_{N_{1}}, \tilde{\eta}_{1}, \ldots \eta_{N_{2}}\right)$ where $\eta_{1}<\cdots<\eta_{N_{1}}<0<\tilde{\eta}_{1}<\cdots<\eta_{N_{2}}$ and $\eta_{s} \simeq \zeta_{1}, \tilde{\eta}_{s^{\prime}} \simeq \zeta_{2}$ for $s=1 \ldots N_{1}, s^{\prime}=1 \ldots N_{2}$. The equivariant $\chi$-genus is then given by a suitable residue of

$$
\begin{aligned}
Z_{Q}= & \frac{1}{(y-1 / y)^{N_{1}+N_{2}-1}} \prod_{\substack{s, s^{\prime}=1 \ldots N_{1} \\
s \neq s^{\prime}}} \frac{u_{s^{\prime}}-u_{s}}{u_{s} / y-y u_{s^{\prime}}} \prod_{\substack{s . s^{\prime}=1 \ldots N_{2} \\
s \neq s^{\prime}}} \frac{v_{s^{\prime}}-v_{s}}{v_{s} / y-y v_{s^{\prime}}} \\
& \times \prod_{\substack{s=1 \ldots N_{1} \\
s^{\prime}=1 \ldots N_{2}}} \prod_{A=1}^{m} \frac{y \nu_{A} v_{s^{\prime}}-u_{s} / y}{u_{s}-\nu_{A} v_{s^{\prime}}} \prod_{s=1 \ldots N_{1}} \frac{\mathrm{d} u_{s}}{\prod_{s}} \prod_{\substack{s^{\prime}=1 \ldots N_{2} \\
s_{s^{\prime}}}}^{\mathrm{d} v_{s^{\prime}}}
\end{aligned}
$$

For $\left(N_{1}, N_{2}\right)$ coprime, it turns out that residues involving the vector multiplet hyperplanes $u_{s} / y-y u_{s^{\prime}}, v_{s} / y-y v_{s^{\prime}}$ vanish. For generic fugacities, the intersections of the chiral multiplet hyperplanes are non-degenerate, and in one-to-one correspondence with bipartite trees linking $N_{1}$ 'black' vertices $e_{1}, \ldots e_{N_{1}}$ to $N_{2}$ 'white' vertices $f_{1}, \ldots, f_{N_{2}}$, with edges $e_{s}-f_{s^{\prime}}$ colored by an integer $A \in\{1, \ldots, m\}$. Each edge represents one hyperplane $u_{s}-\nu_{A} v_{s^{\prime}}$, and each tree corresponds to a particular non-degenerate intersection, which may or may not carry a stable flag. As usual, stable flags correspond to fixed points of the toric action $\phi_{A s s^{\prime}} \mapsto \nu_{A} \phi_{A s s^{\prime}}$ on the space of solutions to the D-term equations (2.1), up to gauge transformations. Since the quiver moduli space is compact, the dependence on $\theta_{A}$ must cancel after summing over all fixed points. In the limit $y \rightarrow 1$, each stable flag contributes a rational number $\pm 1 /\left(N_{1} ! N_{2}\right.$ !), which must add up to the Euler characteristic which is integer. Alternatively, by assuming that the $\theta_{A}$ 's are hierarchically ordered, e.g. $\theta_{1} \ll \theta_{2} \ll \cdots \ll \theta_{m}$, one may associate a given power $\pm y^{2 J_{3}} /\left(N_{1} ! N_{2} !\right)$ to each fixed point, which must add up to the $\chi$-genus.

\subsubsection{Rank $(2,3)$}

For illustration, we consider the rank $(2,3)$ case in some detail. For generic values of the fugacities, one finds contributions from two types of graphs, depicted in figure 2:

- $m(m-1)(m-2)$ flags of type $F_{A B C C}$ with $A, B, C$ all distinct,

$$
F_{1}=\left(-u_{1}+\nu_{A} v_{3},-u_{1}+\nu_{B} v_{2},-u_{1}+\nu_{C} v_{1},-u_{2}+\nu_{A} v_{3}\right)
$$

contributing $-\frac{1}{12}$ as $y \rightarrow 1$. 


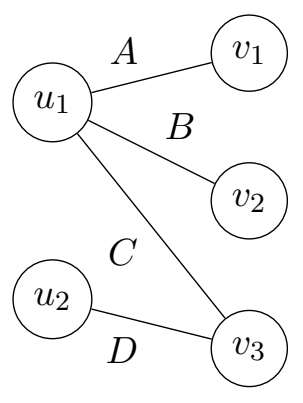

$F_{A B C D}$

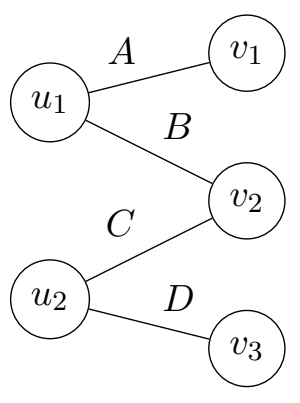

$W_{A B C D}$

Figure 2. Graphs associated to the flags contributing to the equivariant $\chi$-genus of the Kronecker quiver $K_{m}(2,3)$ (up to permutations of $\left\{u_{s}\right\}$ and $\left\{v_{s^{\prime}}\right\}$ )

- $3 \times m(m-1)(m-2)$ flags of type $F_{A B C C}$ with $A, B, C$ all distinct,

$$
\begin{aligned}
& F_{2}=\left(-u_{2}+\nu_{A} v_{3},-u_{1}+\nu_{B} v_{2},-u_{1}+\nu_{C} v_{1},-u_{1}+\nu_{A} v_{3}\right) \\
& F_{3}=\left(-u_{1}+\nu_{C} v_{3},-u_{2}+\nu_{A} v_{2},-u_{1}+\nu_{B} v_{1},-u_{1}+\nu_{A} v_{2}\right) \\
& F_{4}=\left(-u_{1}+\nu_{C} v_{3},-u_{1}+\nu_{B} v_{2},-u_{2}+\nu_{A} v_{1},-u_{1}+\nu_{A} v_{1}\right)
\end{aligned}
$$

contributing $+\frac{1}{12}$ as $y \rightarrow 1$; note that $F_{1}$ and $F_{2}$ only differ by the order of the hyperplanes, while $F_{2,3,4}$ differ by a permutation of $\left\{v_{1}, v_{2}, v_{3}\right\}$.

- $6 \times m(m-1)^{3}$ flags of type $W_{A B C D}$ with $A \neq B, B \neq C, C \neq D$,

$$
\begin{aligned}
& W_{1}=\left(-u_{2}+\nu_{A} v_{3},-u_{1}+\nu_{D} v_{1},-u_{1}+\nu_{C} v_{2},-u_{2}+\nu_{B} v_{2}\right) \\
& W_{2}=\left(-u_{2}+\nu_{A} v_{3},-u_{1}+\nu_{D} v_{2},-u_{1}+\nu_{C} v_{1},-u_{2}+\nu_{B} v_{1}\right) \\
& W_{3}=\left(-u_{2}+\nu_{A} v_{2},-u_{1}+\nu_{D} v_{1},-u_{1}+\nu_{C} v_{3},-u_{2}+\nu_{B} v_{3}\right) \\
& W_{4}=\left(-u_{1}+\nu_{D} v_{3},-u_{2}+\nu_{A} v_{1},-u_{1}+\nu_{C} v_{2},-u_{2}+\nu_{B} v_{2}\right) \\
& W_{5}=\left(-u_{1}+\nu_{D} v_{2},-u_{2}+\nu_{A} v_{1},-u_{1}+\nu_{C} v_{3},-u_{2}+\nu_{B} v_{3}\right) \\
& W_{6}=\left(-u_{1}+\nu_{D} v_{3},-u_{2}+\nu_{A} v_{2},-u_{1}+\nu_{C} v_{1},-u_{2}+\nu_{B} v_{1}\right)
\end{aligned}
$$

contributing $+\frac{1}{12}$ as $y \rightarrow 1$. These flags differ by permutations of $\left\{u_{1}, u_{2}\right\}$ and $\left\{v_{1}, v_{2}, v_{3}\right\}$.

In total, we thus find $2 m\left(3 m^{3}-7 m^{2}+3 m+1\right)$ stable flags, contributing as $y \rightarrow 1$

$$
\begin{aligned}
m(m-1)(m-2) \times \frac{-1}{12}+3 \times m(m & -1)(m-2) \times \frac{1}{12} \\
+ & 6 \times m(m-1)^{3} \times \frac{1}{12}=\frac{m^{4}}{2}-\frac{4 m^{3}}{3}+m^{2}-\frac{m}{6}
\end{aligned}
$$

More generally, for $y \neq 1$ we find

$$
\chi_{Q}=\frac{y^{6 m-4}+y^{4-6 m}+(y+1 / y)^{4}-\left(y^{6}+3 y^{4}+3 y^{2}+2\right) y^{2 m-4}-\left(2 y^{6}+3 y^{4}+3 y^{2}+1\right) y^{-2-2 m}}{(y-1 / y)^{4}(y+1 / y)^{2}\left(y^{2}+1+y^{-2}\right)}
$$

in agreement with Reineke's formula. 
In the absence of flavor fugacities, the non-degenerate intersections collide into a single degenerate intersection involving all chiral multiplet hyperplanes. We find that 4 stable flags contribute, corresponding to $F_{1}, F_{2}, F_{3}, F_{4}$ above with $\nu_{A}=1$, with the flags $F_{2,3,4}$ producing equal contributions. As an example, for $m=3$ we find

$$
\begin{aligned}
-\frac{1}{2}\left(\frac{7}{y^{6}}+\frac{27}{y^{4}}+\frac{55}{y^{2}}+69+\right. & \left.55 y^{2}+27 y^{4}+7 y^{6}\right) \\
& +\frac{3}{6}\left(\frac{9}{y^{6}}+\frac{29}{y^{4}}+\frac{61}{y^{2}}+75+61 y^{2}+29 y^{4}+9 y^{6}\right) \\
=\frac{1}{y^{6}}+\frac{1}{y^{4}}+\frac{3}{y^{2}}+3+3 y^{2}+y^{4}+y^{6} & \stackrel{y \rightarrow 1}{\longrightarrow}-\frac{247}{2}+3 \times \frac{91}{2}=13
\end{aligned}
$$

again in agreement with (5.30).

Let us now apply the Cauchy-Bose identity for the $\mathrm{U}(2)$ vector multiplets, keeping the fugacities generic. In this case, the same non-degenerate intersections contribute separately to each of the two partitions $(1+1,2)$ :

- Each of the $m(m-1)(m-2)$ flags of type $F_{1}$ contribute $\left(\frac{1}{4},-\frac{1}{3}\right)$

- Each of the $3 \times m(m-1)(m-2)$ flags of type $F_{2,3,4}$ contribute $\left(0, \frac{1}{12}\right)$

- Each of the $6 \times m(m-1)^{3}$ flags of type $W_{1, \ldots 6}$ contribute $\left(\frac{1}{12}, 0\right)$

- In addition, there are $3 \times 2 \times m(m-1)$ flags of type $F_{A A B B}$ with $A \neq B$ which contribute $\left(\frac{1}{12},-\frac{1}{12}\right)$

After summing these contributions, we arrive at the result predicted by the MPS formula (2.10) with $S=\{1\}$,

$$
\chi_{K_{m}(2,3)}=\frac{y-y^{-1}}{2\left(y^{2}-y^{-2}\right)} \chi_{K_{2 m}(1,3)}+\frac{1}{2} \chi_{S_{m, m}(3,1,1)}
$$

where $K_{2 m}$ is the Kronecker quiver with $2 m$ arrows, while $S_{m, m}$ is the star quiver considered in section 5.2 for $a_{1}=a_{2}=m$. Similarly, applying the Cauchy-Bose identity for the U(3) vector multiplets, one finds

$$
\chi_{K_{m}(2,3)}=\frac{y-y^{-1}}{3\left(y^{3}-y^{-3}\right)} \chi_{K_{3 m}(2,1)}+\frac{y-y^{-1}}{2\left(y^{2}-y^{-2}\right)} \chi_{S_{2 m, m}(2,1,1)}+\frac{1}{6} \chi_{S_{m, m, m}(2,1,1,1)}
$$

in agreement with the MPS formula (2.10) with $S=\{2\}$.

Finally, let us apply the Cauchy-Bose identity for both the $\mathrm{U}(2)$ and $\mathrm{U}(3)$ vector multiplets. We find contributions from the 6 possible partitions $\lambda=\left(\lambda_{1}, \lambda_{2}\right)$ of the dimension vector $(2,3)$ :

- For $\lambda=(2,3)$, we find $m$ flags of type $F_{A A A A}$ contributing $-\frac{2}{3}$ each, and $3 m$ flags of type $F_{A A A A}$ contributing $\frac{1}{6}$, leading to $\chi_{Q}^{\lambda}=-m / 6$ in the limit $y \rightarrow 1$;

- For $\lambda=(2,2+1)$, we find $m^{2}$ flags of type $F_{A A B B}$ contributing +1 , and $2 m^{2}$ flags of type $F_{A A B B}$ contributing $-\frac{1}{4}$, leading to $\chi_{Q}^{\lambda}=m^{2} / 2$; 
- For $\lambda=(2,1+1+1)$, we find $m^{3}$ flags of type $F_{A B C C}$ contributing $-\frac{1}{3}$, leading to $\chi_{Q}^{\lambda}=-m^{3} / 2$;

- For $\lambda=(1+1,3)$, we find $m^{2}$ flags of type $F_{A A A B}$ contributing $\frac{1}{2}$, leading to $\chi_{Q}^{\lambda}=$ $m^{2} / 2$

- For $\lambda=(1+1,2+1)$, we find $2 m(m-1)$ flags of type $F_{A A A B}$ with $A \neq B$ and $m$ flags of type $F_{A A A A}$, each contributing $-\frac{3}{4} ; 2 m(m-1)$ flags of type $F_{A A A B}$ with $A \neq B$ and $m$ flags of type $F_{A A A A}$, each contributing $-\frac{1}{4}$; and $2 m(m-1)^{2}$ flags of type $W_{A A B C}$ with $A \neq B, B \neq C$, each contributing $-\frac{1}{2}$; in total, $\chi_{Q}^{\lambda}=-m^{3}$;

- For $\lambda=(1+1,1+1+1)$, we find $m^{3}$ flags of type $F_{A B C C}$ contributing $\frac{1}{4} ; m(m-1)$ flags of type $F_{A A A B}$ contributing $\frac{1}{4} ; 3 m^{3}$ flags of type $F_{A B C C}$ plus $3 m(m-1)$ flags of type $F_{A A A B}$ with $A \neq B$, each contributing $\frac{1}{12} ; 6 m(m-1)^{2}$ flags of type $W_{A A B C}$ with $A \neq B, B \neq C$ contributing $\frac{1}{6} ; 6 m(m-1)^{3}$ flags of type $W_{A B C D}$ with $A \neq$ $B, B \neq C, C \neq D$ contributing $\frac{1}{12}$; in total, $\chi_{Q}^{\lambda}=m^{4} / 2$;

These results are summarized in the table below, retaining the dependence on $y$ :

\begin{tabular}{|c|c|c|}
\hline$\lambda$ & $\chi_{Q}^{\lambda}$ & $y \rightarrow 1$ \\
\hline$(2,3)$ & $-\kappa(6 m) \rho_{2} \rho_{3}$ & $-m / 6$ \\
$(2,2+1)$ & $\kappa(2 m) \kappa(4 m) \rho_{2}^{2}$ & $m^{2} / 2$ \\
$(2,1+1+1)$ & $-\frac{1}{6} \kappa(2 m)^{3} \rho_{2}$ & $-m^{3} / 3$ \\
$(1+1,3)$ & $\frac{1}{2} \kappa(3 m)^{2} \rho_{3}$ & $m^{2} / 2$ \\
$(1+1,2+1)$ & $-\frac{1}{2} \kappa(m) \kappa(2 m)(\kappa(3 m)+\kappa(m)) \rho_{2}$ & $-m^{3}$ \\
$(1+1,1+1+1)$ & $\frac{1}{12} \kappa(m)^{3}(\kappa(3 m)+3 \kappa(m))$ & $m^{4} / 2$ \\
\hline
\end{tabular}

where $\rho_{k}=(y-1 / y) / k /\left(y^{k}-y^{-k}\right)$ and $\kappa(m)=(-1)^{m}\left(y^{m}-y^{-m}\right) /(y-1 / y)$. The contributions of the various partitions perfectly match the result of the Coulomb branch formula, which in this case follows from the wall-crossing formula [24, (A.4)] (changing $m \rightarrow-m$ in this equation, and setting $\bar{\Omega}\left(N_{1}, N_{2}\right)=0$ unless $\left.N_{1} N_{2}=0\right)$

$$
\begin{aligned}
\chi_{K_{m}(2,3)}= & -\kappa(6 m) \bar{\Omega}(2,0) \bar{\Omega}(0,3)+\frac{1}{2}[\kappa(3 m)]^{2} \bar{\Omega}(1,0)^{2} \bar{\Omega}(0,3) \\
& +\kappa(2 m) \kappa(4 m) \bar{\Omega}(2,0) \bar{\Omega}(0,2) \bar{\Omega}(0,1) \\
& -\frac{1}{2}\left[\kappa(m)^{2} \kappa(2 m)+\kappa(m) \kappa(2 m) \kappa(3 m)\right] \bar{\Omega}(1,0)^{2} \bar{\Omega}(0,2) \bar{\Omega}(0,1) \\
& -\frac{1}{6} \kappa(2 m)^{3} \bar{\Omega}(2,0) \bar{\Omega}(0,1)^{3}+\frac{1}{2}\left[3 \kappa(m)^{4}+\kappa(m)^{3} \kappa(3 m)\right] \bar{\Omega}(1,0)^{2} \bar{\Omega}(0,1)^{3}
\end{aligned}
$$

where $\bar{\Omega}(k, 0)=\bar{\Omega}(0, k)=\rho_{k}$.

\subsubsection{Rank $(2,2)$}

In the case where the dimension vector is not primitive, there can be contributions from singularities involving vector multiplet hyperplanes. As a result, the contributions are still 
classified by trees but they are no longer bipartite, since they can involve edges of the form $e_{s}-e_{s^{\prime}}$ or $f_{s}-f_{s^{\prime}}$ corresponding to the hyperplanes $u_{s} / y-y u_{s^{\prime}}$ or $v_{s} / y-y v_{s^{\prime}}$. As an example, we consider the rank $(2,2)$ case. In the absence of flavor fugacities, we find that among the 21 singularities, three intersections support stable flags with non-trivial contributions. As an example, for $m=3$ we get

\begin{tabular}{|c|c|}
\hline flag & residue \\
\hline$F=\left(v_{2}-u_{1}, v_{1}-u_{1}, v_{2}-u_{2}\right)$ & $-\frac{1}{2}\left(\frac{2}{y^{5}}+\frac{11}{y^{3}}+\frac{20}{y}+20 y+11 y^{3}+2 y^{5}\right)$ \\
$G_{1}=\left(v_{1}-u_{1}, v_{2}-u_{2}, u_{2}-y^{2} u_{1}\right)$ & $\frac{10+27 y^{2}+37 y^{4}+27 y^{6}+10 y^{8}}{4 y^{3}\left(1+y^{2}\right)}$ \\
$G_{2}=\left(v_{2}-u_{1}, v_{1}-u_{2}, u_{2}-y^{2} u_{1}\right)$ & $\frac{10+27 y^{2}+37 y^{4}+27 y^{6}+10 y^{8}}{4 y^{3}\left(1+y^{2}\right)}$ \\
\hline
\end{tabular}

Adding up these terms, we find

$$
\bar{\chi}_{K_{3}(2,2)}=-\frac{\left(2+3 y^{2}+2 y^{4}\right)\left(1+y^{4}+y^{8}\right)}{2 y^{5}\left(1+y^{2}\right)} \stackrel{y \rightarrow 1}{\rightarrow}-\frac{21}{4}
$$

in agreement with Reineke's formula's for the rational invariant,

$$
\bar{\chi}_{K_{m}(2,2)}=-\frac{\left(y^{m}-y^{-m}\right)\left(2 y^{3 m-2}+2 y^{2-3 m}-2 y^{m+2}-2 y^{-2-m}-y^{3}-y^{-3}+y+y^{-1}\right)}{2(y-1 / y)^{3}(y+1 / y)^{2}} \stackrel{y \rightarrow 1}{\rightarrow} \frac{-m^{3}}{2}+m^{2}-\frac{m}{4}
$$

For generic flavor fugacities, the singularities become non-degenerate. We find that the following stable flags contribute: (see figure 3)

- $2 \times m(m-1)^{2}$ flags of type $F_{A B C}$ with $A \neq B, B \neq C$,

$$
\begin{aligned}
& F_{1}=\left(-u_{1}+\nu_{B} v_{2},-u_{1}+\nu_{A} v_{1},-u_{2}+\nu_{C} v_{2}\right) \\
& F_{2}=\left(-u_{1}+\nu_{A} v_{1},-u_{2}+\nu_{C} v_{2},-u_{1}+\nu_{B} v_{2}\right)
\end{aligned}
$$

contributing $-\frac{1}{4}$ as $y \rightarrow 1$;

- $2 m$ flags of type $G_{A}$,

$$
\begin{aligned}
& G_{1}=\left(-u_{1}+\nu_{A} v_{1},-u_{2}+\nu_{A} v_{2},-u_{1} y^{2}+u_{2}\right) \\
& G_{2}=\left(-u_{1}+\nu_{A} v_{2},-u_{2}+\nu_{A} v_{1},-u_{1} y^{2}+u_{2}\right)
\end{aligned}
$$

contributing $\frac{1}{8}$ in the limit $y \rightarrow 1$ as $y \rightarrow 1$;

In total, we get

$$
\bar{\chi}_{K_{m}(2,2)}=2 m(m-1)^{2} \times-\frac{1}{4}+2 m \times \frac{1}{8}=-\frac{m^{3}}{2}+m^{2}-\frac{m}{4}
$$

in agreement with [32, §6.3].

Applying the Cauchy-Bose formula for both nodes, this result decomposes as a sum over partitions $\lambda=\left(\lambda_{1}, \lambda_{2}\right)$ of the dimension vector $(2,2)$ : 


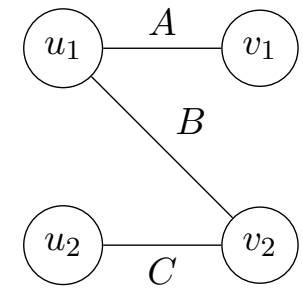

$F_{A B C}$

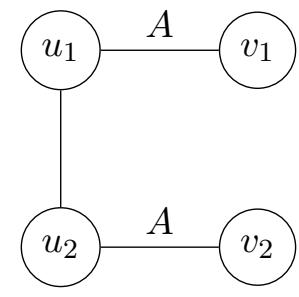

$G_{A}$

Figure 3. Graphs associated to the flags contributing to the equivariant $\chi$-genus of the Kronecker quiver $K_{m}(2,2)$ (up to permutations of $\left\{u_{s}\right\}$ and $\left\{v_{s^{\prime}}\right\}$ )

- For $\lambda=(2,2)$, we find $m$ flags of type $F_{A A A}$ contributing $-\frac{1}{2}$ each and $2 m$ flags of type $G_{A}$ contributing $-\frac{1}{8}$ each, leading to $\chi_{Q}^{\lambda}=-\frac{m}{4}$;

- For $\lambda=(2,1+1)$ or $\lambda=(1+1,2)$, we find $m^{2}$ flags of type $F_{A A B}$ contributing $\frac{1}{2}$ each, leading to $\chi_{Q}^{\lambda}=\frac{m^{2}}{2}$;

- For $\lambda=(1+1,1+1)$, we find $2 m(m-1)$ flags of type $F_{A A B}$ with $A \neq B$ and $m$ flags of type $F_{A A A}$, contributing $-\frac{1}{2}$ each; and $2 m(m-1)^{2}$ flags of type $F_{A B C}$ with $A \neq B \neq C ;$ in total $\chi_{Q}^{\lambda}=-\frac{m^{3}}{2} ;$

These results are summarized in the table below, retaining the dependence on $y$ :

\begin{tabular}{|c|c|c|}
\hline$\lambda$ & $\chi_{Q}^{\lambda}$ & $y \rightarrow 1$ \\
\hline$(2,2)$ & $\kappa(4 m) \rho_{2}^{2}$ & $-m / 4$ \\
$(2,1+1)$ & $\frac{1}{2}[\kappa(2 m)]^{2} \rho_{2}$ & $m^{2} / 2$ \\
$(1+1,2)$ & $\frac{1}{2}[\kappa(2 m)]^{2} \rho_{2}$ & $m^{2} / 2$ \\
$(1+1,1+1)$ & $\frac{1}{4} \kappa(m)^{2} \kappa(2 m)$ & $-m^{3} / 2$ \\
\hline
\end{tabular}

The contributions of the various partitions match the result of the Coulomb branch formula $[24,($ A.4)]

$$
\begin{aligned}
\bar{\chi}_{K_{m}(2,2)}= & -\kappa(4 m) \bar{\Omega}(2,0) \bar{\Omega}(0,2)+\frac{1}{2}[\kappa(2 m)]^{2} \bar{\Omega}(1,0)^{2} \bar{\Omega}(0,2) \\
& +\frac{1}{2}[\kappa(2 m)]^{2} \bar{\Omega}(2,0) \bar{\Omega}(0,1)^{2}-\frac{1}{4} \kappa(m)^{2} \kappa(2 m) \bar{\Omega}(1,0)^{2} \bar{\Omega}(0,1)^{2}
\end{aligned}
$$

where $\bar{\Omega}(k, 0)=\bar{\Omega}(0, k)=\rho_{k}$.

\subsection{Non-abelian quivers with oriented loops}

Finally, we turn to an example of non-abelian quiver with oriented loops. In this case, applying the Cauchy-Bose formula to all nodes leads to a sum over partitions of the total dimension vector as a sum of multiples of basis vectors $\ell \alpha_{a}$, whereas the Coulomb branch formula also includes contributions of single-centered invariants involving combinations of these basis vectors which support an oriented loop. Upon matching the two formulae, we find that the single-centered invariants naturally decompose into contributions from different partitions, whose mathematical meaning remains to elucidate. 
To exhibit this phenomenon, let us consider the simple case of a 3-node cyclic quiver $C_{a, b, c}$ with $a>0, b>0, c>0$ and dimension vector $(2,1,1)$. In order to compare with the analysis in Sec 6.1 of [17] (up to a cyclic permutation of the nodes), we assume

$$
\begin{array}{rrrrr}
b<2 a, & c<a, & k=b+2(c-a)>0, & \\
\zeta_{2}>0, & \zeta_{2}+\zeta_{3}>0, & \zeta_{3} & <0, & \zeta_{1} \rightarrow 0^{-}
\end{array}
$$

which includes the attractor point $\zeta^{*}=(c-a, 2 a-b, b-2 c)$. Simple choices of $(a, b, c)$ satisfying these conditions are

$$
(4,7,3),(5,7,4),(5,8,4),(6,7,5),(6,8,5),(7,7,6),(7,8,6),(8,7,7),(8,8,7), \ldots
$$

We upgrade the stability vector $\zeta$ into $\left(\eta_{0}, \eta_{1} ; \eta_{2}, \eta_{3}\right)=\left(\zeta_{1}-\epsilon, \zeta_{1}+\epsilon, \zeta_{2}, \zeta_{3}\right)$ with $0<\epsilon \ll$ $\left|\zeta_{1}\right|$, and choose the R-charges to be $R_{12}=R_{23}=R_{31}=2 / 3$ so as to allow for a generic superpotential. Denoting the Cartan variables by $\left(v_{1}, v_{2} ; u_{1}, u_{2}\right)$, the $\chi$-genus of the quiver moduli space is given by a sum of residues of

$$
\begin{aligned}
Z_{Q}= & \frac{1}{2(y-1 / y)^{3}} \frac{\left(v_{1}-v_{2}\right)\left(v_{2}-v_{1}\right)}{\left(v_{2} / y-v_{1} y\right)\left(v_{1} / y-v_{2} y\right)} \times\left(\frac{y^{1 / 3} u_{2}-u_{1} / y}{u_{1}-y^{-2 / 3} u_{2}}\right)^{b} \\
& \times \prod_{s=1,2}\left(\frac{y^{1 / 3} u_{1}-v_{s} / y}{v_{s}-y^{-2 / 3} u_{1}}\right)^{a}\left(\frac{y^{1 / 3} v_{s}-u_{2} / y}{u_{2}-y^{-2 / 3} v_{s}}\right)^{c} \frac{\mathrm{d} u_{1}}{u_{1}} \frac{\mathrm{d} u_{2}}{u_{2}} \frac{\mathrm{d} v_{1}}{v_{1}}
\end{aligned}
$$

Out of the 15 singular points, 12 intersections are non-degenerate and three are degenerate. Out of those three, one is projective and two are not, but have $\operatorname{det} \kappa=0$ so can be safely ignored. For the stability conditions above, the only contributing stable flag is $\left(u_{1}-y^{-2 / 3} u_{2}, u_{2}-y^{-2 / 3} v_{1}, u_{2}-y^{-2 / 3} v_{2}\right)$, producing a symmetric Laurent polynomial of degree $k-5$, in agreement with the complex dimension of $\mathcal{M}_{Q}$. On the other hand, the Coulomb branch formula in this chamber predicts

$$
\begin{aligned}
\chi_{Q}= & \Omega_{\mathrm{S}}(2,1,1)+\left(\frac{y-1 / y}{2\left(y^{2}-1 / y^{2}\right)} g_{C}\left(\left\{2 \gamma_{1}, \gamma_{2}, \gamma_{3}\right\}\right)+H\left(\left\{\gamma_{1}, \gamma_{2}, \gamma_{3}\right\},\{2,1,1\}\right)\right) \\
& +\left(\frac{1}{2} g_{C}\left(\left\{\gamma_{1}, \gamma_{1}, \gamma_{2}, \gamma_{3}\right\}\right)+H\left(\left\{\gamma_{1}, \gamma_{1}, \gamma_{2}, \gamma_{3}\right\},\{1,1,1,1\}\right)\right)
\end{aligned}
$$

where $\Omega_{\mathrm{S}}(2,1,1)$ is the single-centered invariant, and the modified Coulomb indices are given by $[17, \S 6.1]$

$$
\begin{aligned}
g_{C}\left(\left\{2 \gamma_{1}, \gamma_{2}, \gamma_{3}\right\}\right) & =\frac{(-1)^{k}\left(y^{k}+y^{-k}\right)}{(y-1 / y)^{2}} \\
H\left(\left\{\gamma_{1}, \gamma_{2}, \gamma_{3}\right\},\{1,1,2\}\right) & =\left\{\begin{array}{c}
\frac{1}{4}\left(y-y^{-1}\right)^{-2}\left(y+y^{-1}\right)^{-1} \\
\times\left\{-\left(y+y^{-1}\right)^{2}+(-1)^{k / 2}\left(y-y^{-1}\right)^{2}\right\} \\
\frac{1}{2}\left(y-y^{-1}\right)^{-2}
\end{array}\right. \\
g_{C}\left(\left\{\gamma_{1}, \gamma_{1}, \gamma_{2}, \gamma_{3}\right\}\right) & =\frac{(-1)^{k+1}\left(y^{k}-y^{-k}\right)}{(y-1 / y)^{3}} \\
H\left(\left\{\gamma_{1}, \gamma_{2}, \gamma_{3}, \gamma_{3}\right\}\{1,1,1,1\}\right) & =\left\{\begin{array}{l}
\frac{1}{4} k\left(y-y^{-1}\right)^{-2}\left(y+y^{-1}\right) \\
-\frac{1}{2} k\left(y-y^{-1}\right)^{-2}
\end{array}\right.
\end{aligned}
$$



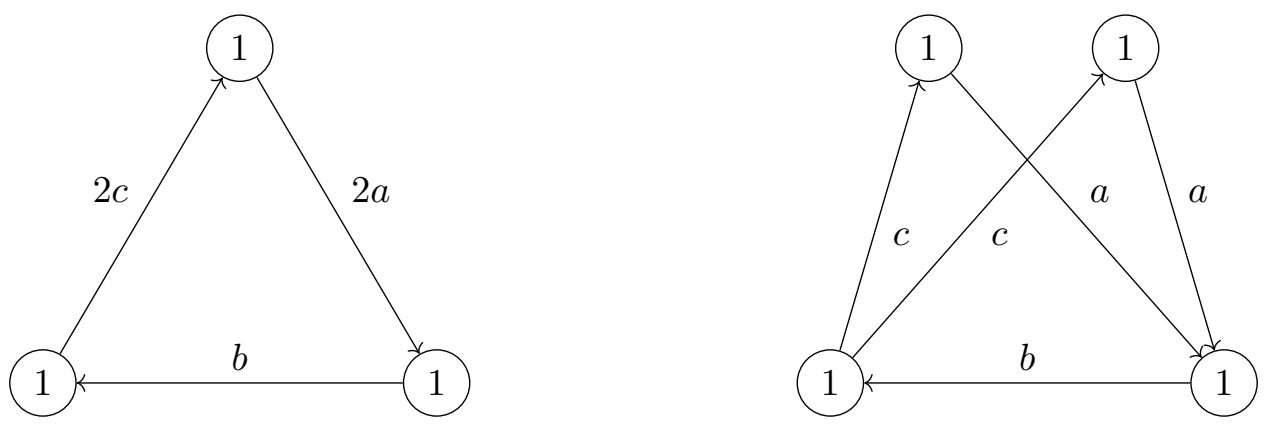

Figure 4. Abelian quivers rising in the MPS formula for the 3-node quiver with rank $(2,1,1)$.

where the two options correspond to $k=b+2(c-a)$ even and odd. respectively. By comparing the result from the residue formula with (5.47), we can read off the singlecentered invariant $\Omega_{\mathrm{S}}(2,1,1)$, e.g. for $(a, b, c)=(7,8,6)$ we get

$$
\Omega(2,1,1)=1862(y+1 / y) \quad \rightarrow \quad \Omega_{\mathrm{S}}(2,1,1)=1863(y+1 / y)
$$

Now, applying the Cauchy-Bose formula for the $\mathrm{U}(2)$ vector multiplets, the $\chi$-index naturally splits into two terms $\chi_{Q}^{(2)}, \chi_{Q}^{(1+1)}$ and associated to the partitions of $N=2$. From the Coulomb branch picture, these two partitions correspond to bound states of charges $\left\{2 \gamma_{1}, \gamma_{2}, \gamma_{3}\right\}$ and $\left\{\gamma_{1}, \gamma_{1}, \gamma_{2}, \gamma_{3}\right\}$, corresponding to the two terms in bracket in (5.47). Thus, it is natural to define 'partial' single-centered invariants $\Omega_{\mathrm{S}}^{(2)}(2,1,1)$ and $\Omega_{\mathrm{S}}^{(1+1)}(2,1,1)$ via

$$
\begin{aligned}
\chi_{Q}^{(2)} & =\Omega_{\mathrm{S}}^{(2)}(2,1,1)+\frac{y-1 / y}{2\left(y^{2}-1 / y^{2}\right)} g_{C}\left(\left\{2 \gamma_{1}, \gamma_{2}, \gamma_{3}\right\}\right)+H\left(\left\{\gamma_{1}, \gamma_{2}, \gamma_{3}\right\},\{2,1,1\}\right) \\
\chi_{Q}^{(1+1)} & =\Omega_{\mathrm{S}}^{(1+1)}(2,1,1)+\frac{1}{2} g_{C}\left(\left\{\gamma_{1}, \gamma_{1}, \gamma_{2}, \gamma_{3}\right\}\right)+H\left(\left\{\gamma_{1}, \gamma_{1}, \gamma_{2}, \gamma_{3}\right\},\{1,1,1,1\}\right)
\end{aligned}
$$

such that $\Omega_{\mathrm{S}}(2,1,1)=\Omega_{\mathrm{S}}^{(2)}(2,1,1)+\Omega_{\mathrm{S}}^{(1,1)}(2,1,1)$. E.g. for $(a, b, c)=(7,8,6)$,

$$
\begin{aligned}
\Omega_{\mathrm{S}}^{(2)}(2,1,1) & =-3(y+1 / y)\left(1903 y^{2}+10976+1903 y^{-2}\right) \\
\Omega_{\mathrm{S}}^{(1+1)}(2,1,1) & =3(y+1 / y)\left(1903 y^{2}+11597+1903 y^{-2}\right)
\end{aligned}
$$

which correctly add up to $1863(y+1 / y)$. One might expect these two contributions to be related to the single-centered invariants of the 3-node and 4-node Abelian quivers obtained by applying the MPS formula (2.10) to the node $V_{1}$ (see figure 4). This expectation is indeed borne out for $\Omega_{\mathrm{S}}^{(1,1)}(2,1,1)$, which is equal to half of the single-centered invariant $\Omega_{\mathrm{S}}(1,1,1,1)$ of the 4-node Abelian quiver, but not for $\Omega_{\mathrm{S}}^{(2)}(2,1,1)$ which appears to be unrelated to the single-centered invariant $\Omega_{\mathrm{S}}(1,1,1)$ associated to the 3 -node Abelian quiver $C_{2 a, b, 2 c}$. It would be interesting to understand the mathematical significance of $\Omega_{\mathrm{S}}^{(2)}(2,1,1)$, or similar 'partial' single-centered invariants arising at higher rank. More generally, it would be of great interest to find a residue prescription (or otherwise) for computing the single-centered invariants $\Omega_{\mathrm{S}}(\gamma, y)$ directly. 


\section{Acknowledgments}

The authors are grateful to M. Reineke and T. Weist for helpful correspondence. S. M. acknowledges the hospitality of LPTHE during part of this project, and the J C Bose Fellowship of Rajesh Gopakumar, from the SERB, Govt. of India. The research of B.P. is supported in part by French state funds managed by the Agence Nationale de la Recherche (ANR) in the context of the LABEX ILP (ANR-11-IDEX-0004-02, ANR-10- LABX-63).

\section{A Implementation of the residue formula in CoulombHiggs.m}

The computations reported in this paper were in part carried out using the Mathematica package CoulombHiggs.m by the last-named author, which was first released along with [37] and has since then been extended to include an implementation of the residue formula (3.1) for general non-Abelian quivers. This package can be freely downloaded from the last author's home page. ${ }^{11}$ Below we briefly outline how to use this package to reproduce some of the computations in this paper, referring to the documentation for more complete information.

Assuming that the file CoulombHiggs.m is present in the user's Mathematica Application directory, the package is loaded by entering

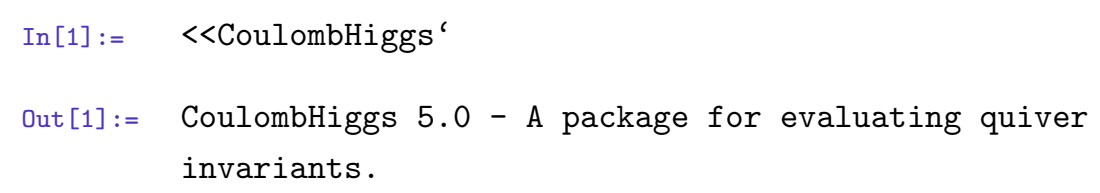

To specify to the Kronecker quiver $K_{3}(2,3)$ considered in section 5.3.1, we first feed in the adjacency matrix, R-charge matrix, stability parameter, dimension vector to the routine JKInitialize:

$\begin{aligned} & \text { In }[2]:=\quad \text { Mat }=\{\{0,-3\},\{3,0\} ; \text { RMat }=0 * \text { Mat; Cvec }=\{-1 / 2,1 / 3\} ; \text { Nvec }=\{2,3\} ; \\ & \text { JKInitialize [Mat, RMat, Cvec, Nvec] ; JKChargeMatrix // } \\ & \text { MatrixForm } \\ & \text { Out }[2]:=\left(\begin{array}{ccccccc}-1 & 0 & 1 & 0 & 0 & 0 & 3 \\ 0 & -1 & 1 & 0 & 0 & 0 & 3 \\ -1 & 0 & 0 & 1 & 0 & 0 & 3 \\ 0 & -1 & 0 & 1 & 0 & 0 & 3 \\ -1 & 0 & 0 & 0 & 1 & 0 & 3 \\ 0 & -1 & 0 & 0 & 1 & 0 & 3\end{array}\right)\end{aligned}$

Among other things, this routine constructs the matrix JKChargeMatrix, whose rows contain the charges of the chiral multiplets under the Cartan algebra $\mathrm{U}(1)^{2} \times \mathrm{U}(1)^{3}$, as well as their R-charge (here, 0) and multiplicity (here, 3); as well as the vector JKEta, which is a small perturbation of $\left(\zeta_{1}, \zeta_{1}, \zeta_{2}, \zeta_{2}, \zeta_{2}\right)$ with increasing entries for each node. The integrand

\footnotetext{
${ }^{11}$ http://www.lpthe.jussieu.fr/ pioline/computing.html
} 
$Z_{Q}$ in (3.2) can be displayed by calling ZTrig[JKChargeMatrix, Nvec], or ZEuler[], ZRational [], ZElliptic [] for (3.3), (3.5) or (3.4), respectively. For computing the index of non-quiver type systems, the charge matrix can be specified by hand.

Having constructed the charge matrix and generalized stability vector, we can then feed these data into the routine JKIndex,

$$
\begin{aligned}
\text { In }[3]:= & \text { JKIndex [JKChargeMatrix, Nvec, JKEta]; JKChiGenus } \\
& 66 \text { stable flags in total; from computing the Euler number, } \\
& 4 \text { stable flags appear to contribute } \\
\text { Out }[3]:= & \left\{-\frac{7 y^{12}+27 y^{10}+55 y^{8}+69 y^{6}+55 y^{4}+27 y^{2}+7}{2 y^{6}},-\frac{-9 y^{12}-29 y^{10}-61 y^{8}-75 y^{6}-61 y^{4}-29 y^{2}-9}{6 y^{6}},\right. \\
& \left.-\frac{-9 y^{12}-29 y^{10}-61 y^{8}-75 y^{6}-61 y^{4}-29 y^{2}-9}{6 y^{6}},-\frac{-9 y^{12}-29 y^{10}-61 y^{8}-75 y^{6}-61 y^{4}-29 y^{2}-9}{6 y^{6}}\right\}
\end{aligned}
$$

Each of the entries in the result, stored in the global variable JKChiGenus, gives the contribution to the $\chi$-genus of those stable flags which contribute non-trivially to the Euler number. In this case, out of 66 stable flags, 4 of those give non-trivial contributions, reproducing the result in (5.31). The relevant flags are stored in the global variable JKRelevantStableFlags,

\begin{aligned} & \hline In $[4]:=\quad$ JKListuDisplay $=\{\mathrm{u} 1, \mathrm{u} 2, \mathrm{v} 1, \mathrm{v} 2, \mathrm{v} 3\} ; \\ &$ DisplayFlagList $[J K R e l e v a n t S t a b l e F l a g s] \\ &$ Out $[4]:=\quad\left(\begin{array}{l}\{0,0,0,0\}\{\mathrm{v} 3-\mathrm{u} 1, \mathrm{v} 2-\mathrm{u} 1, \mathrm{v} 1-\mathrm{u} 1, \mathrm{v} 3-\mathrm{u} 2\}-1 \\ \{0,0,0,0\}\{\mathrm{v} 3-\mathrm{u} 2, \mathrm{v} 2-\mathrm{u} 1, \mathrm{v} 1-\mathrm{u} 1, \mathrm{v} 3-\mathrm{u} 1\}-1 \\ \{0,0,0,0\}\{\mathrm{v} 3-\mathrm{u} 1, \mathrm{v} 2-\mathrm{u} 2, \mathrm{v} 1-\mathrm{u} 1, \mathrm{v} 2-\mathrm{u} 1\}-1 \\ \{0,0,0,0\}\{\mathrm{v} 3-\mathrm{u} 1, \mathrm{v} 2-\mathrm{u} 1, \mathrm{v} 1-\mathrm{u} 2, \mathrm{v} 1-\mathrm{u} 1\}-1\end{array}\right)\end{aligned}$

where the first entry in each row corresponds to the intersection point (dropping the coordinate along the frozen coordinate $u_{1}$, specified by the variable JKFrozenCartan defined by JKInitialize), the second entry to the ordered list of hyperplanes defining each flag $F$, and the last entry to $\operatorname{sgn}\left(\operatorname{det} \kappa_{F}\right)$. The covering tree associated to each flag can also be displayed by using DisplayFlagTree,

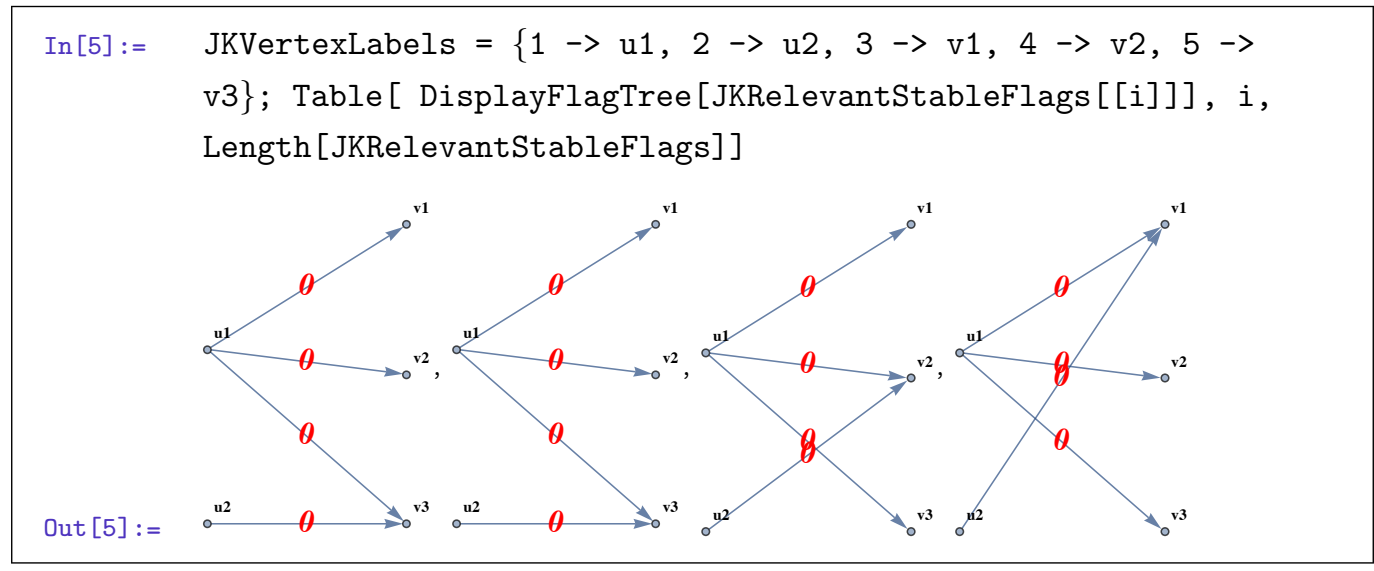


The red zeros on each arrow indicate half the R-charge, which vanishes in this example. Flavor fugacities can be switched on by defining

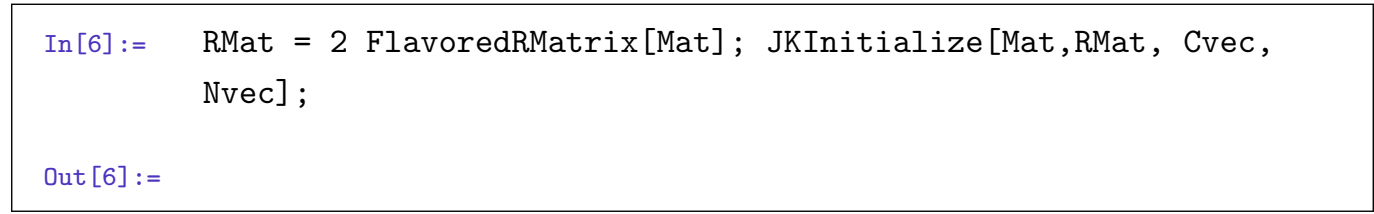

The global variable JKChargeMatrix now includes $2 \times 3 \times 3$ rows, each of which with R-charge $2 \operatorname{th}[A]$ where th $[A]$ are generic fugacity parameters. Running JKIndex will now produce 1410 stable flags, out of which 168 contribute a non-zero residue as in (5.29).

Finally, the same computation can be carried out using the Cauchy-Bose formula for either, or both factors of the gauge group $\mathrm{U}(2) \times \mathrm{U}(3)$. This is done using JKIndexSplit, which takes the same arguments as JKIndex plus the subset $S \subset\{1,2\}$ as in (2.10). E.g. the results reported above (5.32) can be verified by using

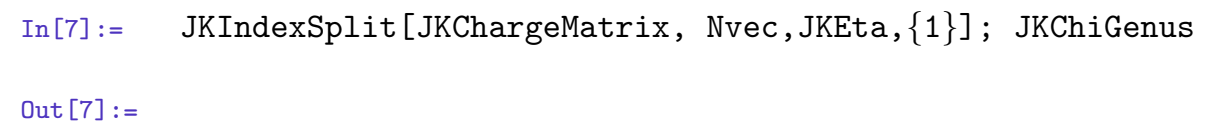

and studying the structure of the relevant flags in JKRelevantStableFlags.

Open Access. This article is distributed under the terms of the Creative Commons Attribution License (CC-BY 4.0), which permits any use, distribution and reproduction in any medium, provided the original author(s) and source are credited.

\section{References}

[1] F. Denef, Supergravity flows and D-brane stability, JHEP 08 (2000) 050 [hep-th/0005049] [INSPIRE].

[2] B. Bates and F. Denef, Exact solutions for supersymmetric stationary black hole composites, JHEP 11 (2011) 127 [hep-th/0304094] [INSPIRE].

[3] K.-M. Lee and P. Yi, Dyons in $N=4$ supersymmetric theories and three pronged strings, Phys. Rev. D 58 (1998) 066005 [hep-th/9804174] [INSPIRE].

[4] F. Denef, Attractors at weak gravity, Nucl. Phys. B 547 (1999) 201 [hep-th/9812049] [INSPIRE].

[5] D. Bak, C.-k. Lee, K.-M. Lee and P. Yi, Low-energy dynamics for 1/4 BPS dyons, Phys. Rev. D 61 (2000) 025001 [hep-th/9906119] [INSPIRE].

[6] J.P. Gauntlett, N. Kim, J. Park and P. Yi, Monopole dynamics and BPS dyons $N=2$ superYang-Mills theories, Phys. Rev. D 61 (2000) 125012 [hep-th/9912082] [INSPIRE].

[7] F. Denef, Quantum quivers and Hall/hole halos, JHEP 10 (2002) 023 [hep-th/0206072] [INSPIRE].

[8] S. Lee and P. Yi, Framed BPS states, moduli dynamics and wall-crossing, JHEP 04 (2011) 098 [arXiv: 1102.1729] [INSPIRE]. 
[9] H. Kim, J. Park, Z. Wang and P. Yi, Ab initio wall-crossing, JHEP 09 (2011) 079 [arXiv: 1107.0723] [INSPIRE].

[10] M.R. Douglas and G.W. Moore, D-branes, quivers and ALE instantons, hep-th/9603167 [INSPIRE].

[11] B. Fiol, The BPS spectrum of $N=2 \mathrm{SU}(N) S Y M$ and parton branes, JHEP 02 (2006) 065 [hep-th/0012079] [INSPIRE].

[12] B. Fiol and M. Mariño, BPS states and algebras from quivers, JHEP 07 (2000) 031 [hep-th/0006189] [INSPIRE].

[13] M. Alim et al., BPS quivers and spectra of complete $N=2$ quantum field theories, Commun. Math. Phys. 323 (2013) 1185 [arXiv:1109.4941] [INSPIRE].

[14] M. Alim et al., $\mathcal{N}=2$ quantum field theories and their BPS quivers, Adv. Theor. Math. Phys. 18 (2014) 27 [arXiv:1112.3984] [INSPIRE].

[15] S. Cecotti, The quiver approach to the BPS spectrum of a $4 d N=2$ gauge theory, Proc. Symp. Pure Math. 90 (2015) 3 [arXiv:1212.3431] [InSPIRE].

[16] M. Reineke, Moduli of representations of quivers, Congr. Rep., Eur. Math. Soc., Zürich 20 (2008) 589 [arXiv:0802.2147].

[17] J. Manschot, B. Pioline and A. Sen, From black holes to quivers, JHEP 11 (2012) 023 [arXiv: 1207.2230] [INSPIRE].

[18] S.-J. Lee, Z.-L. Wang and P. Yi, BPS states, refined indices and quiver invariants, JHEP 10 (2012) 094 [arXiv:1207.0821] [INSPIRE].

[19] M. Kontsevich and Y. Soibelman, Stability structures, motivic Donaldson-Thomas invariants and cluster transformations, arXiv:0811.2435 [INSPIRE].

[20] D. Joyce and Y. Song, A theory of generalized Donaldson-Thomas invariants, arXiv:0810.5645 [INSPIRE].

[21] D. Joyce, Generalized Donaldson-Thomas invariants, arXiv:0910.0105 [INSPIRE].

[22] F. Denef and G.W. Moore, Split states, entropy enigmas, holes and halos, JHEP 11 (2011) 129 [hep-th/0702146] [INSPIRE].

[23] E. Andriyash, F. Denef, D.L. Jafferis and G.W. Moore, Wall-crossing from supersymmetric galaxies, JHEP 01 (2012) 115 [arXiv:1008.0030] [INSPIRE].

[24] J. Manschot, B. Pioline and A. Sen, Wall crossing from Boltzmann black hole halos, JHEP 07 (2011) 059 [arXiv: 1011.1258] [INSPIRE].

[25] M. Reineke, The Harder-Narasimhan system in quantum groups and cohomology of quiver moduli, Invent. Math. 152 (2003) 349.

[26] A.D. King and C.H. Walter, On Chow rings of fine moduli spaces of modules, J. Reine Angew. Math. 461 (1995) 179.

[27] F. Benini, R. Eager, K. Hori and Y. Tachikawa, Elliptic genera of two-dimensional $N=2$ gauge theories with rank-one gauge groups, Lett. Math. Phys. 104 (2014) 465 [arXiv: 1305.0533] [INSPIRE].

[28] F. Benini, R. Eager, K. Hori and Y. Tachikawa, Elliptic genera of $2 d \mathcal{N}=2$ gauge theories, Commun. Math. Phys. 333 (2015) 1241 [arXiv:1308.4896] [INSPIRE]. 
[29] E. Witten, Two-dimensional gauge theories revisited, J. Geom. Phys. 9 (1992) 303 [hep-th/9204083] [INSPIRE].

[30] G.W. Moore, N. Nekrasov and S. Shatashvili, D particle bound states and generalized instantons, Commun. Math. Phys. 209 (2000) 77 [hep-th/9803265] [InSPIRE].

[31] K. Hori, H. Kim and P. Yi, Witten index and wall crossing, JHEP 01 (2015) 124 [arXiv: 1407.2567] [INSPIRE].

[32] K. Ohta and Y. Sasai, Exact results in quiver quantum mechanics and BPS bound state counting, JHEP 11 (2014) 123 [arXiv:1408.0582] [INSPIRE].

[33] C. Cordova and S.-H. Shao, An index formula for supersymmetric quantum mechanics, arXiv: 1406.7853 [INSPIRE].

[34] L.C. Jeffrey and F.C. Kirwan, Localization for nonabelian group actions, Topology 34 (1995) 291.

[35] A. Szenes and M. Vergne, Toric reduction and a conjecture of Batyrev and Materov, Inv. Math. 158 (2004) 453 [math/0306311].

[36] J. Manschot, B. Pioline and A. Sen, A fixed point formula for the index of multi-centered $N=2$ black holes, JHEP 05 (2011) 057 [arXiv:1103.1887] [INSPIRE].

[37] J. Manschot, B. Pioline and A. Sen, On the Coulomb and Higgs branch formulae for multi-centered black holes and quiver invariants, JHEP 05 (2013) 166 [arXiv:1302.5498] [INSPIRE].

[38] J. Manschot, B. Pioline and A. Sen, Generalized quiver mutations and single-centered indices, JHEP 01 (2014) 050 [arXiv:1309.7053] [INSPIRE].

[39] I. Bena et al., Scaling BPS solutions and pure-Higgs states, JHEP 11 (2012) 171 [arXiv: 1205.5023] [INSPIRE].

[40] S.-J. Lee, Z.-L. Wang and P. Yi, Quiver invariants from intrinsic Higgs states, JHEP 07 (2012) 169 [arXiv:1205.6511] [INSPIRE].

[41] J. Manschot, B. Pioline and A. Sen, The Coulomb branch formula for quiver moduli spaces, arXiv:1404.7154 [INSPIRE].

[42] M. Reineke, J. Stoppa and T. Weist, MPS degeneration formula for quiver moduli and refined GW/Kronecker correspondence, Geom. Topol. 16 (2012) 2097.

[43] V.A. Kazakov, I.K. Kostov and N.A. Nekrasov, D particles, matrix integrals and KP hierarchy, Nucl. Phys. B 557 (1999) 413 [hep-th/9810035] [INSPIRE].

[44] M. Mariño and P. Putrov, ABJM theory as a Fermi gas, J. Stat. Mech. 1203 (2012) P03001 [arXiv:1110.4066] [INSPIRE].

[45] S.-J. Lee, Z.-L. Wang and P. Yi, Abelianization of BPS quivers and the refined Higgs index, JHEP 02 (2014) 047 [arXiv: 1310.1265] [INSPIRE].

[46] H. Derksen and J. Weyman, Quiver representations, Notices AMS 52 (2005) 200.

[47] J. de Boer, S. El-Showk, I. Messamah and D. Van den Bleeken, Quantizing $N=2$ multicenter solutions, JHEP 05 (2009) 002 [arXiv:0807.4556] [INSPIRE].

[48] M.F. Atiyah and R. Bott, A Lefschetz fixed point formula for elliptic complexes: II, Ann. Math. 88 (1968) 450. 
[49] S. Alexandrov and B. Pioline, Attractor flow trees, BPS indices and quivers, arXiv: 1804.06928 [INSPIRE].

[50] G. Frobenius, Uber die elliptischen funktionen zweiter art, J. Reine Angew. Math. 93 (1882) 53.

[51] C. Cordova and S.-H. Shao, Counting trees in supersymmetric quantum mechanics, Ann. Inst. H. Poincaré Comb. Phys. Interact. 5 (2018) 1 [arXiv:1502.08050] [InSPIRE].

[52] H. Kim, Scaling behaviour of quiver quantum mechanics, JHEP 07 (2015) 079 [arXiv: 1503.02623] [INSPIRE].

[53] T. Weist, Localization in quiver moduli spaces, Repr. Theor. AMS 17 (2013) 382 [arXiv: 0903.5442].

[54] S. Mozgovoy and M. Reineke, Abelian quiver invariants and marginal wall-crossing, Lett. Math. Phys. 104 (2014) 495 [arXiv:1212.0410].

[55] C. Cordova and S.-H. Shao, Asymptotics of ground state degeneracies in quiver quantum mechanics, Commun. Num. Theor. Phys. 10 (2016) 339 [arXiv:1503.03178] [inSPIRE]. 